\title{
Methodology for Increasing the Efficiency of Dynamic Process Calculations in Elastic Elements of Complex Engineering Constructions
}

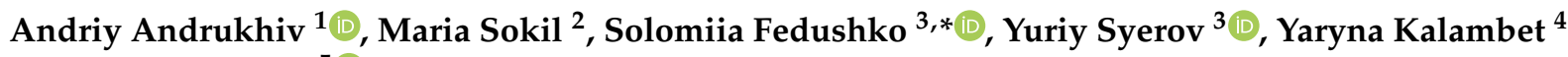 \\ and Tomas Peracek ${ }^{5}$ (D) \\ 1 Scientific and Technical Library, Lviv Polytechnic National University, 79007 Lviv, Ukraine; \\ andriy.i.andrukhiv@lpnu.ua \\ 2 Department of Transport Technologies, Institute of Engineering Mechanics and Transport, Lviv Polytechnic \\ National University, 79007 Lviv, Ukraine; mariia.b.sokil@lpnu.ua \\ 3 Department of Social Communication and Information Activities, Institute of the Humanities and Social \\ Sciences, Lviv Polytechnic National University, 79007 Lviv, Ukraine; yurii.o.sierov@lpnu.ua \\ 4 Department of Philology, Interpreting and Translation, Institute of Humanities and Public Administration, \\ Ivano-Frankivsk National Technical University of Oil and Gas, 76019 Ivano-Frankivsk, Ukraine; \\ yarynakalambet@gmail.com \\ 5 Department of Information Systems, Faculty of Management, Comenius University in Bratislava, \\ 82005 Bratislava, Slovakia; tomas.peracek@fm.uniba.sk \\ * Correspondence: solomiia.s.fedushko@lpnu.ua
}

check for updates

Citation: Andrukhiv, A.; Sokil, M.; Fedushko, S.; Syerov, Y.; Kalambet, Y.; Peracek, T. Methodology for Increasing the Efficiency of Dynamic Process Calculations in Elastic Elements of Complex Engineering Constructions. Electronics 2021, 10, 40 https://doi.org/10.3390/electronics 10010040

Received: 27 November 2020 Accepted: 22 December 2020 Published: 29 December 2020

Publisher's Note: MDPI stays neutral with regard to jurisdictional clai$\mathrm{ms}$ in published maps and institutional affiliations.

Copyright: $(\odot 2020$ by the authors. Licensee MDPI, Basel, Switzerland. This article is an open access article distributed under the terms and conditions of the Creative Commons Attribution (CC BY) license (https:// creativecommons.org/licenses/by/ $4.0 /)$.

\begin{abstract}
This study deals with a methodology for increasing the efficiency of dynamic process calculations in elastic elements of complex engineering constructions. We studied the complex dynamic processes in a simple engineering construction, a mechanical system of an elastic bodycontinuous flow of homogeneous medium. The developed methodology is based on the use of a priori information on some of the vibrations forms, the construction of a "simplified" mathematical model of system dynamics, and the obtaining of an analytical relationship that describe the overall range of factors on the elastic vibrations of system. The methodology is used for cases of complex vibrations of elastic bodies, and the obtained results can serve as a basis for choosing the main technological and operational parameters of elastic elements of mechanisms and machines that perform complex vibrations. The results obtained in this work are the basis for calculating the blast effect on the elements of protective structures in order to increase their protective capacity by improving the method of their attachment or by using additional reinforcement, buff load effects on the elements of drilling strings and dynamic processes that occur during surface strengthening by work hardening in order to avoid resonance phenomena, and technological processes of vibration displacement or vibration separation of granular media.
\end{abstract}

Keywords: dynamic processes; simplest engineering construction; continuous flow; homogeneous medium; complex vibration; priori information; mathematical model

\section{Introduction}

A methodology for researching complex vibrations of the structural elements of the simplest engineering constructions has been developed. It is based on the usage of a priori information about one of the vibration forms of an elastic body, its processing and usage during the construction of a mathematical model of another form of vibrations, and development or usage of existing analytical methods for constructing a solution for the latter one. The methodology, however, simplifies the mathematical model of process dynamics and thus simplifies the procedure of finding and researching its solution. Using this methodology enables one to establish a number of features of the structural elements' dynamics, in particular the conditions of external and internal resonances and their initial 
peculiarities. The main idea of the methodology is presented using the example of an elastic body that performs bending and longitudinal vibrations, and in addition to that a continuous flow of homogeneous medium (CFHM) of zero stiffness that moves along the body, which greatly complicates the problem of constructing a solution for the mathematical model of elastic body dynamics. For this reason, the following issues were predetermined:

- even movement at a constant speed along the elastic body of the CFHM affects the natural frequencies of the component vibrations;

- the most dangerous (resonant) vibrations of the specified type of systems can be caused both by external periodic forces and mutual influence of certain forms of vibrations on the others (internal resonances);

- internal resonances of an elastic body that performs bending and longitudinal vibrations can exist with even and odd modes of bending vibrations; with even modes they are caused by its longitudinal vibrations and with odd modes by its longitudinal vibrations and continuous flow of homogeneous medium motion;

- the amplitude of bending vibrations of the transition through the internal resonance at higher frequencies of longitudinal vibrations is less than at the second frequency;

- the amplitude of the transition through the main resonance for larger values of the amount of motion of the continuous flow of homogeneous medium is smaller than the amplitude of the transition during the rapid transition through the resonance.

The methodology can be generalized in other cases of complex vibrations of elastic bodies, and the obtained results can serve as the basis for choosing the main technological and operational parameters of elastic elements of mechanisms and machines that perform complex vibrations. The reliability of the obtained calculated dependences is confirmed by the boundary transition from which follow the results known from scientific publications concerning the one form of vibrations of elastic bodies.

\section{Problem Statement and Research Relevance}

Analysis of dynamic processes in the elements of machines and structures, which are elastic bodies, shows that in most cases they carry out such complex oscillations through a combination of longitudinal forces, bending, and torsion. Isolation during research of some of them, even the largest amplitude with simultaneous neglect of the influence of others (even small amplitude) will lead to significant inaccuracies in describing the dynamic process of the element and the system as a whole. The value of the error increases significantly in cases where the natural frequencies of the oscillations are close in value or related by a rational ratio, and the elastic properties of the object under study are described by nonlinear relations.

The analytical study of complex nonlinear dynamic processes in elastic bodies (construction elements) based on mathematical models that take into account a wide range of external and internal factors is associated with significant mathematical difficulties. This is the integration of systems of nonlinear non-autonomous boundary value problems for equations with partial derivatives. Therefore, there is an actual problem of development of such an analytical methodology that takes into account a broad range of factors that impact on the object and receive its calculation-dependent base that would be available for engineering calculations at the design and upgrade stage. A partial solution for these problems is illustrated with the case of an elastic element mechanism (construction), which provides both longitudinal and bending vibrations. In addition, the elastic element is characterized by longitudinal frequencies, or it moves along the continuous flow of an incompressible homogeneous environment.

The study of even its monoform nonlinear oscillations is a difficult mathematical problem, because it is impossible to use existing analytical methods of partial differential equations to solve it.

To partially resolve the problem, an analytical and empirical method is developed. Its main idea includes the following tasks: based on empirical information on one of the forms of oscillations (usually of smaller amplitude), the approximate analytical relations 
of their amplitude-frequency characteristics are obtained; the obtained dependences are taken into account in the refined mathematical model of another form of oscillations. The latter non-autonomous type is much simpler to study than the "idealized model". One of the most convenient analytical methods is used for this model, or a new approach is developed, for example, for systems with strong nonlinearity.

\section{Related Works}

The most effective methods of analytical study of nonlinear oscillatory processes of systems with concentrated masses and distributed parameters are those based on the main idea of perturbation methods [1,2]. They are effectively used to study single or multifrequency oscillations of elastic bodies, as well as systems with distributed parameters $[3,4]$. However, the operation of existing structures is usually accompanied by complex oscillations of their elements. For example, turbine rotors perform simultaneous bending and torsional oscillations [5,6], columns for drilling oil and gas wells perform both longitudinal bending and torsional oscillations $[7,8]$, etc. Some experimental and theoretical studies show that components of vibrations of structural elements, in some cases interacting with each other, can lead to significant dynamic loads in them. These loads increase rapidly in magnitude in the so-called resonant cases, when the frequencies of the component oscillations (bending, longitudinal, torsional) or external and natural frequencies are related by rational relations [2,9] (i.e., oscillations in bodies with material properties that are or are nearly linearly elastic). To prevent them means to extend the service life of elastic bodies, i.e., structures as a whole, only on the basis of mathematical models adequate to the dynamic process and their solutions. If such phenomena under the action of external perturbation for both quasilinear and nonlinear systems with concentrated masses have found a relatively proper development (see, for example [1,9]), the issues of internal resonances for systems with distributed parameters were considered only in some works $[9,10]$. To prevent such phenomena, it is necessary to know in advance about the reaction of the object under study to a particular type of disturbance, as well as the result of the interaction of certain types of oscillations. However, the development of analytical methods for appropriate mathematical models that take into account a wide range of external and internal factors is a complex problem. The use of the same for the latter methods of numerical simulation in many cases does not give the desired results. The problem is much more complicated for elastic elements whose elastic properties of the material are described by nonlinear relations [11-15] or are characterized by longitudinal velocity [16-22]. The peculiarity of the dynamics of the last class of systems is an example of this fact. The velocity of the relative motion of the medium along the elastic body (or the body itself) affects the quantitative characteristics of the components of oscillations (longitudinal or bending), and thus the second component of oscillations (torsional). On the other hand, taking into account the constant velocity of longitudinal motion in the mathematical model of monoform oscillations of elastic bodies leads to a qualitatively new form for which it is not possible to apply known classical methods of integrating equations with partial derivatives $[16,17,20,21]$. Taken together, it requires the development of simplified methods for studying this class of systems and obtaining relatively simple analytical dependencies that could predict in advance such undesirable conditions for the resonant phenomena of the class of bodies (systems).

Thus, the paper attempts to partially solve such a little-covered problem in the literature as the development of methods for analytical research of complex nonlinear oscillations of the simplest elements of machines and mechanisms. Its main idea is illustrated by the example of nonlinear elastic bodies that carry both bending and longitudinal vibrations besides the body that moves along a continuous flow in a homogenous environment. Such systems include, in particular, columns for drilling wells along which a continuous flow of fluid moves, elements of pipelines for transporting liquids, working bodies of screw conveyors for moving bulk media $[7,19,23]$, and others. They are the main focus of the work. Regarding the external perturbation of the oscillations of an elastic body, the cases 
of continuous and discrete action are considered in the work. The latter is analytically represented by delta functions [24-31]. The resonance condition for the discrete action of pulsed perturbation is obtained, and on the basis of generalization of the main idea of asymptotic methods [29-31] of nonlinear mechanics on systems with pulsed perturbation, the laws of change of basic parameters of resonant oscillations are described.

\section{Materials and Methods}

The main idea of the developed methodology for studying complex nonlinear elastic body oscillations is presented below on the example of the elastic body-CFHM system, which moves along a body with a constant velocity $V$. The elastic body under the action of external factors performs complex vibrations, known as bending and longitudinal vibrations. Partial information about one of the forms of vibrations, in this case longitudinal, which is known from experimental studies, is considered in experimental studies based on the processing of information obtained by the use of sensors. Regarding these specified data, the amplitude of these oscillations is much smaller than the bending. Despite this fact, the following vibrations under certain conditions can lead to a significant increase in the amplitude of another form of bending vibrations of the body and thus reduce the service life. The task is to:

(a) develop methods for processing experimental information about longitudinal vibrations and bringing them to a form convenient for consideration when building a mathematical model of bending vibrations;

(b) construct a mathematical model of the dynamics of the elastic body-CFHM system, which would take into account the above a priori information and a wide range of internal and external factors of continuous and discrete action;

(c) develop methods for constructing the solution of the above mathematical model;

(d) obtain analytical relations that describe the defining parameters of bending vibrations of an elastic body depending on external and internal factors of the system on the basis of the above method. That would enable calculations of specific elements of machines or systems at the design stage or during their operation.

Methods of processing experimental information about one of the forms of vibrations and bringing it to a form convenient for building a mathematical model of another form of vibrations. The peculiarity of the elastic body dynamics is the fact that its material satisfies the quasilinear elastic properties, and therefore the frequency of the dynamic process of such an elastic body depends on the amplitude. It is proposed to use a priori information about the amplitude and period of longitudinal vibrations of an elastic body to establish this relationship with respect to longitudinal vibrations [14]. Thus, it is believed that it is determined by a set of successive values of amplitudes and periods of vibrations. These characteristics of the longitudinal vibrations of a rigid body determine the following approximate ratios:

$$
\frac{\Delta b}{\tau}=\frac{d b}{d t}, \frac{\Delta \tau}{\tau}=\frac{d \tau}{d t}
$$

in which $\Delta b=b_{j+1}-b_{j}, \Delta \tau=\tau_{j+1}-\tau_{j}, \Delta \tau$-length of $j$-scope, $b_{j}$-magnitude.

Logarithms of amplitude change $\delta_{b}=\ln \frac{b_{j}}{b_{j+1}}$ and period $\delta_{\tau}=\ln \frac{\tau_{j}}{\tau_{j+1}}$ of vibrations with a sufficient degree of accuracy can be approximated by polynomials

$$
\delta_{b}=\beta_{1}\left(b_{j+\frac{1}{2}}, \varepsilon\right), \delta_{\tau}=\left[-\bar{\rho}+\beta_{2}\left(b_{j+\frac{1}{2}}, \varepsilon\right)\right] \beta_{1}\left(b_{j+\frac{1}{2}}, \varepsilon\right)
$$

In the above dependences, $\beta_{1}\left(b_{j+\frac{1}{2}}, \varepsilon\right)$ and $\beta_{2}\left(b_{j \frac{1}{2}}, \varepsilon\right)$ polynomials have the form of

$$
\beta_{1}\left(b_{j+\frac{1}{2}}, \varepsilon\right)=\sum_{n} \sum_{k} \varepsilon^{n} \beta_{1 n k} b_{j+\frac{1}{2}}^{2 k}
$$




$$
\beta_{2}\left(b_{j+\frac{1}{2}}, \varepsilon\right)=\sum_{n} \sum_{k} \varepsilon^{n} \beta_{2 n k} b_{j+\frac{1}{2}}^{2 k}
$$

It should be highlighted that in relations (3) and (4), $b_{j+\frac{1}{2}}=\frac{b_{j}+b_{j+1}}{2}, \bar{\rho}$ is a constant, which is expressed in terms of physical and mechanical properties of the elastic body, the method of its fixation and for the quasilinear properties of the latter is zero, a small parameter indicating a slow change and time of amplitude and period of vibrations.

From the expressions for the logarithms of the change in the amplitude and period of vibrations on the basis of the mean theorem we obtain

$$
\begin{gathered}
\int_{\tau_{j}}^{\tau_{j+1}} \frac{d}{d t}\left(\beta_{1}\left(b_{j+\frac{1}{2}}, \varepsilon\right)\right) d t=\tau_{j}\left(\frac{d}{d t} \ln b\right)_{\mid b=b_{j+\frac{1}{2}}} \\
\int_{\tau_{j}}^{\tau_{j+1}} \frac{d}{d t}\left(\beta_{2}\left(b_{j+\frac{1}{2}}, \varepsilon\right)\right) d t=\tau_{j}\left(\frac{d}{d t} \ln \tau\right)_{\mid \tau=j+\frac{1}{2}}, \\
2 \pi=\psi_{j+1}-\psi_{j}=\tau_{j}\left(\frac{d \psi}{d t}\right)_{\mid \tau=\tau_{j+\frac{1}{2}}}
\end{gathered}
$$

where $\psi$ is phase of the longitudinal component of vibrations.

Regarding the obtained expressions to the characteristics of the vibrating process at a given time, we obtain

$$
\frac{d b}{d t}=\sum_{n} \varepsilon^{n} b \hat{A}_{n}(b), \frac{d \psi}{d t}=\omega b^{\bar{\rho}}+\sum_{n} \varepsilon^{n} \hat{B}_{n}(b),
$$

where $\hat{A}_{n}(b), \hat{B}_{n}(b)$ are known functions.

According to the above, the longitudinal movement of the cross section of the elastic body with the coordinate $\mathrm{y}$ for the first approximation can be represented as $u(y, t)=$ $b_{r}(\varepsilon t) \sin \frac{r \pi}{l} y \cos \theta_{r}, \theta_{r}=\left(\omega_{r} t+\phi_{r}\right)(l$ is length of the elastic body and the parameters $r, \omega$ are determined by the method of fixing the body, its physical and mechanical properties and cross-sectional shape).

Mathematical model of elastic body dynamics. As it follows from the formulation of the problem to be solved in the work, an arbitrary conditionally-isolated element of an elastic body, as well as SPOS, are in complex motion. To describe the kinematic parameters of CFHM, portable motion is considered to be the motion of an elastic body. The movement of the continuous flow of homogeneous medium is relative to the body and will be nothing more than a relative motion. As for the elastic body, which performs complex vibrations, bending and longitudinal ones, the first of them will be considered portable, then the longitudinal deformation of the thallus relative to the transversely deformed elastic body determines the relative motion. Assuming that in the undeformed position of the body its neutral axis occupies a position that coincides with the vertical axis OY, provided that the bending vibrations of the body occur in one plane (XOY plane), the portable transverse displacement of the elastic body is uniquely determined by the movement perpendicular to OY. Thus, the transverse vibrations are described by the function-the transverse movement of the neutral axis of the body with the coordinate at any time $t$; the relative displacement of the elastic body (longitudinal vibrations) is unambiguously described by the displacement of the specified cross section of the body along the deformed elastic axis. The specified value is determined, as mentioned above, by the function $u(y, t)$.

A mathematical model of the dynamics of the elastic body system - continuous flow of CFHM can be obtained based on the "dynamic equilibrium" of the conditionally selected element with the coordinate of its middle y and length dy. 
In the case when the elastic body continuous flow of homogeneous medium performs one-form vibrations (bending) at hinged ends, its mathematical model has the following form [11]:

$$
\begin{gathered}
\left(m_{t}+m_{s}\right) \frac{\partial^{2} w}{\partial t^{2}}+2 m_{s} \frac{\partial^{2} w}{\partial y \partial t} V+m_{s} V^{2} \frac{\partial^{2} w}{\partial y^{2}}+E I \frac{\partial^{4} w}{\partial y^{4}}=\varepsilon f_{1}\left(w, \frac{\partial w}{\partial t}, \ldots, \frac{\partial^{3} w}{\partial y^{3}}, \gamma\right) \\
w(y, t)_{\mid y=j}=0,{\frac{\partial^{2} w}{\partial y^{2}}}_{\mid y=j}=0, j=0, l
\end{gathered}
$$

in which $l$ is the distance between the attachment points of the elastic body, $m_{t}, m_{s}$ is accordingly the mass of the unit length of the elastic body and CFHM, EI is its bending stiffness (E-E-modulus, moment of inertia of the body cross-section), $f_{1}\left(w, \frac{\partial w}{\partial t}, \ldots, \frac{\partial^{3} w}{\partial y^{3}}, \gamma\right)$ is a function that describes the resistance forces, the nonlinear component of the reducing force, the external $2 \pi$-periodic $\gamma$ perturbation with frequency $\mu(\gamma=\mu t)$ and other forces whose maximum value is significantly less than the maximum value of the last term of the left side, as indicated by the small parameter $\varepsilon$.

As for the considered much more complex case, the mathematical model should additionally take into account the inertial forces of relative motion and the Coriolis force of an element of an elastic body and CFHM. In accordance with the above-accepted components of the movements of the system under consideration, the relative $\left(\vec{a}_{r}^{t}\right)$ and the Coriolis $\left(\vec{a}_{\text {cor }}^{t}\right)$ acceleration of a point that coincides with the middle of an element of an elastic body (a point with a coordinate $y$ ) is determined by the dependences

$$
\begin{gathered}
\vec{a}_{r}^{t}=\vec{i} \frac{\partial^{2} u}{\partial t^{2}} \sin \alpha+\vec{j} \frac{\partial^{2} u}{\partial t^{2}} \cos \alpha, \\
a_{c o r}^{t}=2\left(\frac{\partial^{2} w}{\partial y \partial t}\right) \vec{k} \times\left(\vec{i} \frac{\partial u}{\partial t} \sin \alpha_{0}+\vec{j} \frac{\partial u}{\partial t} \cos \alpha_{0}\right)=2 \frac{\partial^{2} w}{\partial y \partial t}\left\{-\frac{\partial u}{\partial t} \cos \alpha_{0} \vec{i}+\frac{\partial u}{\partial t} \sin \alpha_{0} \vec{j}\right\}
\end{gathered}
$$

where $\vec{i}, \vec{j}, \vec{k}$ unit vectors are directed along the axes $\mathrm{OX}, \mathrm{OY}$, and $\mathrm{OZ}$ (the $\mathrm{OZ}$ axis is directed perpendicular to the XOY plane); and $\alpha_{0}$ is the angle that is formed by the tangent to the curved axis of the elastic body with the OY axis at the point with the coordinate $y$. For small vibrations of the system (such vibrations are considered in this paper), the condition for determining the specified angle is the correlation $\sin \alpha_{0}=\alpha_{0}=\frac{\partial w}{\partial y}, \cos \alpha=1$, and therefore in accordance with the specified $\frac{\partial^{2} w}{\partial y \partial t}$ is nothing more than the angular velocity of the transpositional motion of elastic body. As for the acceleration of CFHM, for its movement we have the following: the relative acceleration is equal to $\vec{a}_{e}^{s}=\frac{V^{2}}{\rho} \vec{p}$, where $\vec{p}$ is the unit vector of the internal normal to the curved axis of an elastic body, and that is why $\vec{p}=-\vec{i} \cos \alpha_{0}+\vec{j} \sin \alpha_{0}, \rho$-radius of curvature of the neutral axis of the body at a point with an ordinate $y$. Projections of the specified vector on the axis of the fixed system XOYZ are determined in accordance with the correlation

$$
\vec{a}_{e}^{s}=\frac{\partial^{2} w}{\partial t^{2}} \vec{i}+\frac{\partial^{2} u}{\partial t^{2}} \sin \alpha_{0} \vec{i}+\frac{\partial^{2} u}{\partial t^{2}} \cos \alpha_{0} \vec{j}
$$

Similarly, the Coriolis acceleration vector continuous flow of homogeneous medium is determined by

$$
a_{c o r}^{s}=2\left(\frac{\partial^{2} w}{\partial y \partial t}+\frac{\partial^{2} u}{\partial y \partial t}\right) \vec{k} \times V\left(\vec{i} \sin \alpha_{0}+\vec{j} \cos \alpha_{0}\right)=2 V\left(\frac{\partial^{2} w}{\partial y \partial t}+\frac{\partial^{2} u}{\partial y \partial t}\right)\left(-\vec{i} \cos \alpha_{0}+\vec{j} \sin \alpha_{0}\right)
$$

in which $\frac{\partial^{2} u}{\partial y \partial t} \vec{k}$ is the vector of angular velocity of an elastic body in relative motion. 
Taken together, the above allows us to write down the differential equation of bending vibrations of an elastic body, taking into account longitudinal vibrations and motion along CFHM in the form of

$$
\begin{gathered}
\left.\left(m_{t}+m_{s}\right) \frac{\partial^{2} w}{\partial t^{2}}+E I \frac{\partial^{4} w}{\partial y^{4}}+m_{s}\left\{2 \frac{\partial^{2} w}{\partial y \partial t} V+V^{2} \frac{\partial^{2} w}{\partial y^{2}}+2 V\left(\frac{\partial^{2} w}{\partial y \partial t}+\frac{\partial^{2} u}{\partial y \partial t}\right)+\frac{\partial^{2} u}{\partial t^{2}} \frac{\partial w}{\partial y}\right)\right\} \\
+m_{t}\left\{\frac{\partial^{2} u}{\partial t^{2}} \frac{\partial w}{\partial y}-2 \frac{\partial^{2} w}{\partial y \partial t} \frac{\partial u}{\partial t}\right\}=f_{1}\left(w, \frac{\partial w}{\partial t}, \ldots, \frac{\partial^{3} w}{\partial y^{3}}, \gamma\right) .
\end{gathered}
$$

Note 1:

(a) Equation (15) takes into account that for the case of small vibrations, it is true that $\sin \alpha_{0}=\alpha_{0}=\frac{\partial w}{\partial y}, \cos \alpha=1$, and the deplanation of flat sections is neglected;

(b) longitudinal vibrations do not change the curvature of the neutral axis of the elastic body;

(c) the differential relation of the "dynamic equilibrium" of the selected element in the projection on the OY axis can, together with Equation (15), serve as a basis for determining the dynamic response of anchorages.

Methodology for studying nonlinear bending vibrations of an elastic body, nonresonant case. As noted above, the main idea of the methodology is based on the use of a priori information for longitudinal vibrations, so the function $u(t, y)$ and its partial derivatives are determined in accordance with the relation obtained on its basis $\left.u(y, t)=b_{r}(\varepsilon t) \sin \frac{r \pi}{l} y \cos \theta_{r}\right)$. Restrictions on the CFHM and magnitude of the amplitude of longitudinal vibrations allow us to represent differential Equation (15) in the form

$$
\left(m_{t}+m_{s}\right) \frac{\partial^{2} w}{\partial t^{2}}+E I \frac{\partial^{4} w}{\partial y^{4}}=\varepsilon f\left(w, \frac{\partial w}{\partial t}, \ldots, \frac{\partial^{3} w}{\partial y^{3}}, \gamma\right)+g\left(\frac{\partial w}{\partial y}, \frac{\partial^{2} w}{\partial y^{2}}, \frac{\partial^{2} w}{\partial y \partial t}, \frac{\partial u}{\partial t}\right)
$$

where

$$
\begin{gathered}
\varepsilon f_{1}\left(w, \frac{\partial w}{\partial t}, \ldots, \frac{\partial^{3} w}{\partial y^{3}}, \gamma\right)=\varepsilon f\left(w, \frac{\partial w}{\partial t}, \ldots, \frac{\partial^{3} w}{\partial y^{3}}, \gamma\right)-m_{s}\left(2 \frac{\partial^{2} w}{\partial y \partial t} V+V^{2} \frac{\partial^{2} w}{\partial y^{2}}\right), \\
\left.g\left(\frac{\partial w}{\partial y}, \frac{\partial^{2} w}{\partial y^{2}}, \frac{\partial^{2} w}{\partial y \partial t}, \frac{\partial u}{\partial t}, \frac{\partial^{2} u}{\partial t^{2}}\right)=-m_{s}\left\{2 V\left(\frac{\partial^{2} w}{\partial y \partial t}+\frac{\partial^{2} w}{\partial y \partial t}\right)+\frac{\partial^{2} u}{\partial t^{2}} \frac{\partial w}{\partial y}\right)\right\}-m_{t}\left\{\frac{\partial^{2} u}{\partial t^{2}} \frac{\partial w}{\partial y}-2 \frac{\partial^{2} w}{\partial y \partial t} \frac{\partial u}{\partial t}\right\}
\end{gathered}
$$
expresses the effect on the bending vibrations of the longitudinal and movements of the CFHM.

It is obvious that the maximum value of the right-hand side of the obtained Equation (16) is a small value compared to the maximum value of the term $E I \frac{\partial^{4} w}{\partial y^{4}}$. This is the basis for applying general ideas for constructing solutions to quasilinear equations with a small right-hand side [15-18] for the boundary value problem (16) and (10). Its peculiarity is that its right part is $2 \pi$-periodic according to two arguments, $\theta$ (takes into account the influence of information known on the basis of experimental information, the influence of longitudinal vibrations on bending ones) and $\gamma$ (takes into account the influence of external perturbation). The solution of Equation (17), more precisely its first approximation in the $s$-form of "dynamic equilibrium", is presented in the form

$$
w(t, y)=a_{s}(t)\left(\sin \left(\chi_{s} y+\psi_{s}(t)\right)+\sin \left(\chi_{s} y-\psi_{s}(t)\right)\right)+\varepsilon U_{1}\left(a_{s}, y, \psi_{s}, \gamma, \theta_{r}\right)
$$

in which $\chi_{s}=\frac{s \pi}{l}$, and $U\left(a_{s}, x, \psi_{s}, \gamma, \theta_{r}\right)$ is an unknown $2 \pi$-periodic function by arguments $\psi_{s}, \gamma, \theta$, which takes into account the influence of external and internal factors on changing the vibrations. As for the law of changes in its parameters $a_{s}(t)$ and $\psi_{s}(t)$, their value significantly depends on the correlation between the frequency of natural bending vibrations $\omega_{s}=\left(\frac{s \pi}{l}\right)^{2} \sqrt{\frac{E I}{m_{t}+m_{s}}}$ on the one hand and the frequency of the external periodic perturbation $\mu$ or longitudinal vibrations $\omega$ on the other hand. In the case when the correlations are justified, $q \omega_{s} \neq p \mu$ та and $q \omega_{s} \neq p \omega_{r}(p, q$ are coprime numbers and this case will be called 
non-resonant) for bending vibrations of the function $a_{S}(t)$ and $\psi_{s}(t)$ in the asymptotic representation (18) are related by ordinary differential equations

$$
\frac{d a_{s}}{d t}=\varepsilon A_{1}\left(a_{s}\right), \cdots \frac{d \psi_{s}}{d t}=\omega_{s}-\left(\chi_{s}\right)^{2} \frac{m_{s}}{m_{t}+m_{s}} \frac{V^{2}}{\omega_{1}}+\varepsilon B_{1}\left(a_{s}\right)
$$

The right-hand sides of relations (19), more precisely unknown functions $A_{1}\left(a_{s}\right)$ and $B_{1}\left(a_{s}\right)$, must be defined so that the asymptotic representation (18), taking into account (19), satisfies the original Equation (17) with the considered accuracy. For an unambiguous definition of the functions $A_{1}\left(a_{s}\right)$ and $B_{1}\left(a_{s}\right)$ on $U\left(a_{s}, y, \psi_{s}, \gamma, \theta\right)$, we impose an additional condition-it does not contain the first mods in the argument scenario. This is equivalent to the following relation:

$$
\int_{0}^{2 \pi} U\left(a_{s}, y, \psi_{s}, \gamma, \theta_{r}\right)\left\{\begin{array}{c}
\sin \psi_{s} \\
\cos \psi_{s}
\end{array}\right\} d \psi_{s}=0
$$

In the physical interpretation, this condition is equivalent to the choice of the s mode amplitude for the bending vibrations amplitude. To find the indeterminate functions $A_{1}\left(a_{s}\right)$ and $B_{1}\left(a_{s}\right)$, which describe the laws of variation of the basic parameters of bending vibrations of an elastic body by differentiating the asymptotic representation of the solution taking into account the relations (19), we obtain

$$
\begin{aligned}
& \frac{\partial w}{\partial t}=\varepsilon A_{1}\left(a_{s}\right)\left(\cos \left(\chi_{s} y+\psi_{s}\right)-\cos \left(\chi_{s} y+\psi_{s}\right)\right)-a_{s}\left(\omega+\varepsilon B_{1}\left(a_{s}\right)\right)\left(\sin \left(\chi_{s} y+\psi_{s}\right)+\sin \left(\chi_{s} y+\psi_{s}\right)\right) \\
& +\varepsilon\left(\omega_{s} \frac{\partial U_{1}\left(a_{s}, x, \psi_{s}, \gamma, \theta_{r}\right)}{\partial \psi_{s}}+\frac{\partial U_{1}\left(a_{s}, x, \psi_{s}, \gamma, \theta_{r}\right)}{\partial \theta_{r}} \omega_{r}+\frac{\partial U_{1}\left(a_{s}, x, \psi_{s}, \gamma, \theta_{r}\right)}{\partial \gamma} \mu\right), \\
& \frac{\partial^{2} u}{\partial t^{2}}=-a_{s} \omega_{s}^{2}\left(\sin \left(\chi_{s} y+\psi_{s}\right)+\sin \left(\chi_{s} y+\psi_{s}\right)\right)-2 \varepsilon\left(A_{1}\left(a_{s}\right)+a_{s} B_{1}\left(a_{s}\right) \omega_{s}\right)\left(\cos \left(\chi_{s} y+\psi_{s}\right)-\cos \left(\chi_{s} y+\psi_{s}\right)\right) \\
& +\varepsilon\left(\frac{\partial^{2} U}{\partial \psi_{s}{ }^{2}} \omega_{1}^{2}+\frac{\partial^{2} U}{\partial \theta_{r}{ }^{2}} \omega_{r}^{2}+\frac{\partial^{2} U}{\partial \gamma^{2}} \mu^{2}+2 \frac{\partial^{2} U}{\partial \psi_{s} \partial \theta_{r}} \omega_{s} \omega_{r}+2 \frac{\partial^{2} U}{\partial \psi_{s} \partial \gamma} \omega_{s} \mu+2 \frac{\partial^{2} U}{\partial \gamma \partial \theta_{r}} \mu \omega_{r}\right), \\
& \frac{\partial^{2} u}{\partial y^{2}}=-a_{s} \chi_{s}^{2}\left(\cos \left(\chi_{s} y+\psi_{s}\right)-\cos \left(\chi_{s} y+\psi_{s}\right)\right)+\varepsilon \frac{\partial^{2} u_{1}}{\partial y^{2}} \\
& \frac{\partial u}{\partial y}=-a_{s} \chi_{s}\left(\sin \left(\chi_{s} y+\psi_{s}\right)-\sin \left(\chi_{s} y-\psi_{s}\right)\right)+\varepsilon \frac{\partial u_{1}}{\partial y} \\
& \frac{\partial^{3} u}{\partial y^{3}}=a_{s} \chi_{s}^{3}\left(\sin \left(\chi_{s} y+\psi_{s}\right)+\sin \left(\chi_{s} y+\psi_{s}\right)\right)+\varepsilon \frac{\partial^{3} u_{1}}{\partial y^{3}}, \\
& \frac{\partial^{4} u}{\partial y^{4}}=a \chi_{s}^{4}\left(\cos \left(\chi_{s} y+\psi_{s}\right)-\cos \left(\chi_{s} y+\psi_{s}\right)\right)+\varepsilon \frac{\partial^{4} u_{1}}{\partial y^{4}} .
\end{aligned}
$$

Thus, we obtain the differential equation for the connection between the indeterminate functions $U_{1}\left(a_{1}, y, \psi_{1}, \gamma, \theta\right)$ and $A_{1}\left(a_{1}\right)$ and $B_{1}\left(a_{1}\right)$ for the first mode of vibrations $(s=1)$.

$$
\begin{gathered}
\left(\omega_{1}\right)^{2} \frac{\partial^{2} U_{1}}{\partial \psi_{1}^{2}}+\omega_{r}^{2} \frac{\partial^{2} U_{1}}{\partial \theta_{r}^{2}}+\mu^{2} \frac{\partial^{2} U_{1}}{\partial \gamma^{2}}+2 \omega_{1} \mu \frac{\partial^{2} U_{1}}{\partial \psi \partial \theta_{r}}+2 \omega_{1} \omega_{r} \frac{\partial^{2} U_{1}}{\partial \psi \partial \theta_{r}}+2 \mu \omega_{r} \frac{\partial^{2} U_{1}}{\partial \gamma \partial \theta_{r}}+\frac{E I}{m_{t}+m_{s}} \frac{\partial^{4} U_{1}}{\partial x^{4}} \\
=\frac{1}{m_{s}+m_{t}} F_{1}\left(a_{1}, y, \psi_{1}, \gamma, \theta_{r}\right)+G_{1}\left(a_{1}, y, \psi_{1}, \theta_{r}\right)+4 A\left(a_{1}\right) \omega_{1} \sin \chi_{1} y \sin \psi_{1}+4 B\left(a_{1}\right) a_{1} \omega_{1} \sin \chi_{1} y \cos \psi_{1}
\end{gathered}
$$

where $\bar{F}_{1}\left(a_{s}, y, \psi_{s}, \gamma\right)$ corresponds to the value $f\left(w, \frac{\partial w}{\partial t}, \ldots, \frac{\partial^{3} w}{\partial y^{3}}, \gamma\right)-m_{s}\left(2 \frac{\partial^{2} w}{\partial y \partial t} V+V^{2} \frac{\partial^{2} w}{\partial y^{2}}\right)$ if the function $w(y, t)$ in it and its derivatives is determined according to the main part in representation (5). As for the function $G_{1}\left(a_{s}, y, \psi_{s}, \theta_{r}\right)$, it, in accordance with the agreed notation, takes approximate values for the considered value as follows. It allows one to obtain the relations to determine the required functions

$$
\begin{gathered}
G_{1}\left(a_{1}, y, \psi_{1}, \theta_{r}\right) \frac{1}{m_{s}+m_{t}}\left\{2 m_{s} V\left(-b_{r} \omega_{r} \frac{r \pi}{l} \cos \frac{r \pi}{l} y \sin \theta_{r}\right) 2 a_{1} m_{t} b_{r} \chi_{1} \omega_{r}^{2} \sin \frac{r \pi}{l} y \cos \theta_{r} \cos \chi_{s} y \cos \psi_{s}\right. \\
\left.\quad-2 a_{1}\left[m_{t} b_{r} \omega_{r} \sin \frac{r \pi}{l} y \sin \theta_{r}\right] \cos \chi_{1} y \sin \psi_{1}\right\} \\
\frac{d a_{1}}{d t}=-\frac{\varepsilon}{6 \pi^{2}\left(m_{t}+m_{s}\right) l \omega_{1}} \int_{0}^{l} \int_{0}^{2 \pi} \int_{0}^{2 \pi} F_{1}\left(a_{1}, y, \psi_{1}, \gamma\right) \sin \chi_{1} y \sin \psi_{1} d y d \psi_{1} d \gamma \\
\quad+\frac{\varepsilon}{6 \pi^{2}\left(m_{t}+m_{s}\right) l \omega_{1}} \int_{0}^{l} \int_{0}^{2 \pi} \int_{0}^{2 \pi} G_{1}\left(a_{1}, y, \psi_{1}, \theta_{r}\right) \sin \chi_{1} y \sin \psi_{1} d y d \psi_{1} d \theta_{r} \\
\frac{\varepsilon}{d t}= \\
\quad \omega_{1}-\frac{\varepsilon}{6 \pi^{2}\left(m_{t}+m_{s}\right) l a_{1} \omega_{1}} \int_{0}^{l} \int_{0}^{2 \pi} \int_{0}^{2 \pi} F_{1}\left(a_{1}, y, \psi_{1}, \gamma\right) \sin \chi_{1} y \cos \psi_{1} d y d \psi_{1} d \gamma+ \\
+\frac{\varepsilon}{6 \pi^{2}\left(m_{t}+m_{s}\right) l a_{1} \omega_{1}} \int_{0}^{l} \int_{0}^{2 \pi} \int_{0}^{2 \pi} G_{1}\left(a_{1}, y, \psi_{1}, \theta_{r}\right) \sin \chi_{1} y \cos \psi_{1} d y d \psi_{1} d \theta_{r} .
\end{gathered}
$$


From the form of the function $G_{1}\left(a_{1}, y, \psi_{1}, \theta_{r}\right)$, it can be seen that

$$
\int_{0}^{l} \int_{0}^{2 \pi} \int_{0}^{2 \pi}\left\{\begin{array}{c}
\cos \psi_{s} \\
\sin \psi_{s}
\end{array}\right\} G_{1}\left(a_{s}, y, \psi_{s}, \theta_{r}\right) \sin \chi_{s} y d y d \psi_{s} d \theta_{r} \equiv 0
$$

The obtained relation allows us to draw the following conclusion: for the first nonresonance approximation, the longitudinal vibrations of the elastic body do not affect the law of change of the single-frequency bending vibrations amplitude. As for the CFHM, it follows from the specified properties of the system that describe the shape of the defected axis of the elastic body, the frequency and amplitude of vibrations. Taken into consideration all the above, the system of differential equations for the first non-resonance approximation (25) can be transformed into

$$
\begin{aligned}
& \frac{d a_{1}}{d t}=-\frac{\varepsilon}{8 \pi^{2}\left(m_{t}+m_{s}\right) l \omega_{1 s}} \int_{0}^{2 \pi} \int_{0}^{2 \pi} \int_{0}^{l} F_{1}\left(a_{1}, y, \psi_{1}, \gamma\right) \sin \chi_{1} y \sin \psi_{1} d y d \psi_{1}=\varepsilon A_{1}\left(a_{1}\right) \\
& \frac{d \psi_{1}}{d t}=\omega_{1}-\frac{m_{s}}{\left(m_{t}+m_{s}\right) \omega_{1}} V^{2}\left(\chi_{1}\right)^{2}-\frac{\varepsilon}{8 \pi^{2}\left(m_{t}+m_{s}\right) l a \omega_{1}} \int_{0}^{2 \pi} \int_{0}^{2 \pi} \int_{0}^{l} F_{1}\left(a_{1}, y, \psi_{1}, \gamma\right) \sin \chi_{1} y \cos \psi_{1} d y d \psi_{1} d \gamma \\
& =\omega_{1}-\frac{m_{s}}{\left(m_{t}+m_{s}\right) \omega_{s}} V^{2}\left(\chi_{1}\right)^{2}+\varepsilon B_{1}\left(a_{1}\right) .
\end{aligned}
$$

For the case when the material of the elastic body satisfies the nonlinear technical law of elasticity [6], and the resistance force is proportional to the speed in the degree $s_{1}$, the right part of Equation (19) takes the form for the case of the main mode of vibrations

$$
A_{1}(a)=v a^{s_{1}}, \varepsilon B_{1}(a)=-\frac{3 k_{2} \varepsilon}{32} \frac{\pi^{2}}{l^{2}} \frac{a^{2}}{\omega_{1}} .
$$

According to the relations (19), taking into account (28) in Figure 1, the laws of change of the natural bending vibrations frequency from the amplitude and speed of the CFHM relative motion (Figure 1a) and the CFHM bulk mass and the length of the elastic element (Figure $1 \mathrm{~b}$ ) at the constant speed of the CFHM relative motion at such parameters of the system $l=8 \mathrm{~m}, m_{t}=30 \mathrm{~kg} / \mathrm{m}, m_{s}=20 \mathrm{~kg} / \mathrm{m}, E=2 \times 10^{12} \mathrm{H} / \mathrm{m}^{2}, I=6 \times 10^{-6} \mathrm{~m}^{4}$ are presented for different values of the CFHM bulk mass.

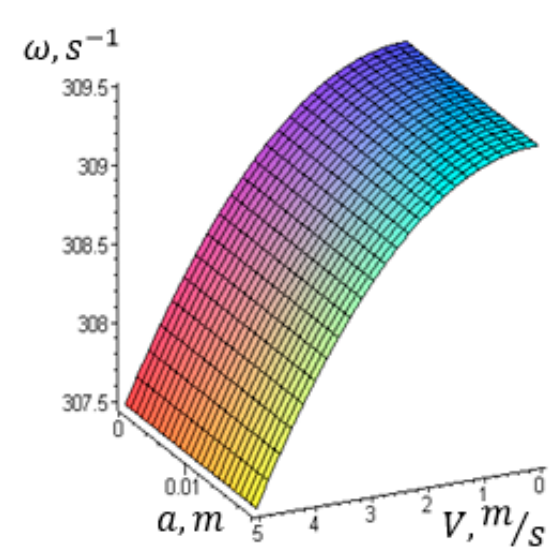

(a)

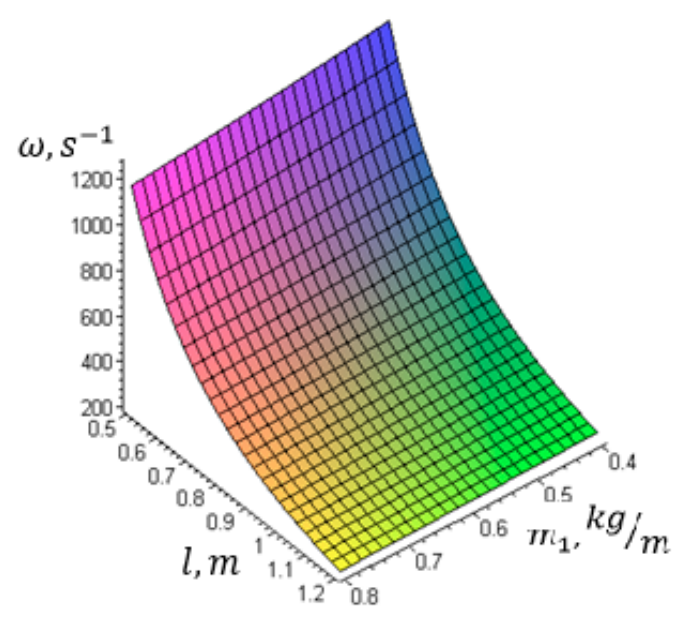

(b)

Figure 1. (a) Relation of the frequency of nonlinear bending vibrations of the elastic body on the amplitude a and continuous flow V of homogeneous medium (CFHM) relative speed V and (b) the length of the elastic body and CFHM bulk mass at a constant relative speed of the latter.

Thus, the influence of nonlinear elastic characteristics of the elastic body material and the motion of a continuous flow of medium along it is manifested as a function of its natural frequency of vibrations on the amplitude and speed of the latter. In particular, the natural frequency of nonlinear vibrations of an elastic body:

- $\quad$ for larger values of the relative flow rate of the continuous medium is less; 
- decreases with increase of elastic body length;

- is smaller for a continuous flow of medium of smaller specific gravity.

The above facts play a significant role in the study of resonance vibrations of the system of elastic body-CFHM continuous flow. These vibrations are the subject of further research.

Methods for studying nonlinear bending vibrations of an elastic body, resonance case. Resonance vibrations almost always play a negative role for mechanical systems, because during resonance they significantly increase the amplitude of vibrations, which means that dynamical loads increase. This reduces the service life of the systems. The peculiarity of the resonance vibrations of the considered elastic body is the fact that they can be caused not only by external forces but also by longitudinal vibrations; in addition, they are affected by the CFHM motion.

In order to find their influence on the resonance values of the amplitude, as in the non-resonance case, we will look for the solution of the problem in the asymptotic representation (18) with the difference that in the resonance case, the pipe vibration's amplitude significantly depends on the phase difference of natural and forced vibrations. First of all, consider the internal resonance. As noted above, it occurs due to longitudinal vibrations, the main parameters of which are known from the processing of experimental studies, and they can be interpreted as external (in relation to bending) action. The phenomenon of resonance in nonlinear systems depends significantly on the phase difference of their natural and coercive forces. For this purpose, we introduce the specified parameter according to the relation $\phi=\psi_{1}-\theta_{r}$, and therefore in the asymptotic representation (18), the amplitude parameter is determined by a more complex relation, namely

$$
\frac{d a}{d t}=\varepsilon A_{1}(a, \phi), \frac{d \phi}{d t}=\omega_{1}-\left(\chi_{1}\right)^{2}-\frac{m_{s}}{m_{t}+m_{s}} \frac{V^{2}}{\omega_{1}}-\frac{q}{p} \omega_{r}+\varepsilon B_{1}(a, \phi) .
$$

Here and below, the index in the amplitude of the main mode of vibrations is omitted for simplicity. The indeterminate functions $A_{1}(a, \phi), B_{1}(a, \phi)$ are located in such a way that the asymptotic representation (18), taking into account (28), satisfies the original equation with the considered degree of accuracy. To do this, by differentiating (18), taking into account the above, we have

$$
\begin{aligned}
\frac{\partial u}{\partial t}= & \varepsilon A_{1}(a, \phi)\left(\cos \left(\chi_{1} y+\phi+\theta_{r}\right)-\cos \left(\chi_{1} y-\left(\phi+\theta_{r}\right)\right)\right) \\
& -a\left(\omega+\mu B_{1}(a, \phi)\right)\left(\sin \left(\chi_{1} y+\phi+\theta_{r}\right)+\sin \left(\chi_{1} y-\left(\phi+\theta_{r}\right)\right)\right)+\frac{q}{p} \omega_{r} \varepsilon \frac{\partial U_{1}}{\partial \theta_{r}}+\omega_{1} \varepsilon \frac{\partial U_{1}}{\partial \psi_{1}} \\
\frac{\partial^{2} u}{\partial t^{2}}= & \varepsilon \frac{\partial A(a, \phi)}{\partial \phi}\left(\omega_{1}-\frac{q}{p} \omega_{r}\right)\left(\cos \left(\chi_{1} y+\phi+\theta_{r}\right)-\cos \left(\chi_{1} y-\left(\phi+\theta_{r}\right)\right)\right) \\
& -\varepsilon \omega A_{1}(a, \phi)\left(\sin \left(\chi_{1} y+\phi+\theta_{r}\right)+\sin \left(\chi_{1} y-(\phi+\theta)\right)\right) \\
& -a\left(\omega^{2}+2 \varepsilon \omega B_{1}\left(a, \chi_{1} y+\phi+\theta_{r}\right)\right)\left(\cos \left(\chi_{1} y+\phi+\theta_{r}\right)-\cos \left(\chi_{1} y-\left(\phi+\theta_{r}\right)\right)\right) \\
& -\varepsilon a \frac{\partial B_{1}(a, \phi)}{\partial \phi}\left(\omega-\frac{q}{p} \omega_{r}\right)\left(\sin \left(\chi_{1} y+\phi+\theta_{r}\right)+\sin \left(\left(\chi_{1} y-\left(\phi+\theta_{r}\right)\right)\right)\right. \\
& +\varepsilon\left(\frac{\partial^{2} U_{1}}{\partial \psi_{1}{ }^{2}} \omega_{1}^{2}+\frac{\partial^{2} U_{1}}{\partial \theta^{2}}\left(\frac{q}{p} \omega_{r}\right)^{2}+\frac{\partial^{2} U_{1}}{\partial \gamma^{2}} \mu^{2}\right. \\
& \left.+2 \frac{\partial^{2} U_{1}}{\partial \partial \partial \psi_{1}} \frac{q}{p} \omega_{r} \omega_{1}+2 \frac{\partial^{2} U_{1}}{\partial \theta \partial} \frac{q}{p} \omega_{r} \mu+2 \frac{\partial^{2} U_{1}}{\partial \gamma \partial \psi_{1}} \mu \omega_{1}\right)
\end{aligned}
$$

The obtained relations allow us to write a differential equation, which connects the required functions in the form

$$
\begin{aligned}
& \omega_{1}^{2} \frac{\partial^{2} U_{1}}{\partial \psi_{1}{ }^{2}}+\left(\frac{q}{p} \omega_{r}\right)^{2} \frac{\partial^{2} U_{1}}{\partial \theta_{r}{ }^{2}}+\mu^{2} \frac{\partial^{2} U_{1}}{\partial \gamma^{2}}+2 \frac{q}{p} \omega_{1} \omega_{r} \frac{\partial^{2} U_{1}}{\partial \psi_{1} \partial \theta_{r}}+2 \omega_{1} \mu \frac{\partial^{2} U_{1}}{\partial \psi \partial \gamma}+2 \frac{q}{p} \mu \omega_{r} \frac{\partial^{2} U_{1}}{\partial \gamma \partial \theta_{r}}+\frac{E I}{m_{t}+m_{s}} \frac{\partial^{4} U_{1}}{\partial y^{4}} \\
& =F(y, a, \psi, \gamma)+a \frac{m_{s}}{m_{t}+m_{s}} V^{2}\left(\frac{\pi}{l}\right)^{2} \sin \frac{\chi \pi}{l} x \cos \psi_{1}+G\left(y, a, \psi, \theta_{r}\right)+ \\
& \sin \frac{\pi y}{l} \times\left(\cos \psi\left(-\frac{\partial A(a, \phi)}{\partial \phi}\left(\omega_{1}-\frac{q}{p} \omega_{r}\right)+2 a \omega_{1} B(a, \phi)\right)+\sin \psi\left(a \frac{\partial B(a, \phi)}{\partial \phi}\left(\omega-\frac{q}{p} \omega_{r}\right)+2 A(a, \phi) \omega\right)\right) .
\end{aligned}
$$

An indeterminate function will satisfy homogeneous boundary conditions if it can be represented as a series

$$
U_{1}(y, a, \psi, \gamma, \theta,)=\sum_{m} \sin \frac{m \pi}{l} y U_{1 m}\left(a, \theta, \psi_{1}, \gamma\right) .
$$


In this case, the functions $A_{1}(a, \phi), B_{1}(a, \phi)$ and their derivatives are connected by a relation 


$$
\begin{aligned}
a V^{2} \frac{m_{s}}{m_{t}+m_{s}}\left(\frac{\pi^{2}}{2 l}\right) \cos \psi_{1} & +\frac{1}{\left(m_{t}+m_{s}\right) l \omega_{s}} \int_{0}^{l} F\left(a, y, \psi_{1}, \theta\right) \sin \frac{\pi}{l} y d y+\frac{1}{l} \int_{0}^{l} G\left(a, y, \psi_{1}, \theta\right) \sin \frac{\pi}{l} y d y \\
& +\left(\cos \psi\left(-\frac{\partial A(a, \phi)}{\partial \phi}\left(\omega-\frac{q}{p} \omega_{r}\right)+2 a \omega B(a, \phi)\right)\right. \\
+ & \left.\sin \psi\left(a \frac{\partial B(a, \phi)}{\partial \phi}\left(\omega-\frac{q}{p} \omega_{r}\right)+2 A(a, \phi) \omega \rightleftarrows\right)\right)=0
\end{aligned}
$$

Relation (33) is the basis for determining the right-hand members of differential Equation (29), which describe the change in the basic parameters of bending vibrations of an elastic body in the resonant zone. Indeed, if $U_{1}\left(a, x, \psi_{1}, \gamma, \theta_{r}\right)$ is $2 \pi$ periodically with an argument $\psi_{1}$, and does not contain in the splitting of its first modes of the splitting, then the coefficients of its splitting for the specified mode of the function $U_{1 m}\left(a, \theta, \psi_{1}, \gamma\right)$ have the same properties. Thus, we obtain a system of equations from relation (33).

$$
\begin{gathered}
a \frac{\partial B(a, \phi)}{\partial \phi}\left(\omega-\frac{q}{p} \omega_{r}\right)+2 A(a, \phi) \omega=\bar{f}_{1}(a)-\frac{1}{\pi} \int_{0}^{l} \int_{0}^{2 \pi} G\left(a, y, \phi+\theta_{r}, \theta_{r}\right) \sin \frac{\pi}{l} y \sin \left(\phi+\theta_{r}\right) d \theta_{r} d y \\
\frac{\partial A(a, \phi)}{\partial \phi}\left(\omega-\frac{q}{p} \omega_{r}\right)-2 a \omega B(a, \phi)=\widetilde{f}_{1}(a)+a V^{2} \frac{m_{s}}{m_{t}+m_{s}}\left(\frac{\pi^{2}}{2 l}\right) \\
-\frac{1}{\pi l} \int_{0}^{l} \int_{0}^{2 \pi} G\left(a, y, \phi+\theta_{r}, \theta_{r}\right) \sin \frac{\pi}{l} y \cos \left(\phi+\theta_{r}\right) d \theta_{r} d y
\end{gathered}
$$

where

$$
\begin{gathered}
\bar{f}_{1}(a)=-\frac{1}{4 \pi^{2}\left(m_{t}+m_{s}\right) l} \int_{0}^{l} \int_{0}^{2 \pi} \int_{0}^{2 \pi} F\left(a, y, \psi_{1}, \theta_{r}\right) \sin \frac{\pi}{l} y \sin \psi d \psi_{1} d \theta_{r} d y, \\
\widetilde{f}_{1}(a)=-\frac{1}{4 \pi^{2}\left(m_{t}+m_{s}\right) l} \int_{0}^{l} \int_{0}^{2 \pi} \int_{0}^{2 \pi} F\left(a, y, \psi_{1}, \theta_{r}\right) \sin \frac{\pi}{l} y \cos d \psi_{1} d \theta_{r} d y .
\end{gathered}
$$

The system of differential Equation (34) allows us to represent equation of the first approximation of resonance vibrations caused by the CFHM motion and longitudinal vibrations in the form

$$
\begin{array}{r}
\frac{d a}{d t}=\varepsilon \bar{f}_{1}(a)+\frac{1}{\pi \omega_{1}} \frac{1}{\pi} \int_{0}^{l} \int_{0}^{2 \pi} G\left(a, y, \phi+\theta_{r}, \theta_{r}\right) \sin \frac{\pi}{l} y \sin \left(\phi+\theta_{r}\right) d \theta_{r} d y \\
\frac{d \phi}{d t}=\omega_{1}-\frac{q}{p} \omega_{r}-a V^{2} \frac{m_{s}}{m_{t}+m_{s}}\left(\frac{\pi^{2}}{2 l}\right)+\varepsilon \widetilde{f}_{1}(a)+\frac{1}{\pi a \omega_{1}} \frac{1}{\pi} \int_{0}^{l} \int_{0}^{2 \pi} G\left(a, y, \phi+\theta_{r}, \theta_{r}\right) \sin \frac{\pi}{l} y \cos \left(\phi+\theta_{r}\right) d \theta_{r} d y \\
\text { here } \\
\bar{f}_{1}(a)=-\frac{1}{4 \pi^{2}\left(m_{t}+m_{s}\right) l} \int_{0}^{l} \int_{0}^{2 \pi} \int_{0}^{2 \pi} F\left(a, y, \psi_{1}, \theta_{r}\right) \sin \frac{\pi}{l} y \sin \psi d \psi_{1} d \theta_{r} d y \\
\tilde{f}_{1}(a)=-\frac{1}{4 \pi^{2}\left(m_{t}+m_{s}\right) l} \int_{0}^{2 \pi} \int_{0}^{2 \pi} \int_{0}^{2 \pi} F\left(a, y, \psi_{1}, \theta_{r}\right) \sin \frac{\pi}{l} y \cos d \psi_{1} d \theta_{r} d y .
\end{array}
$$

Differential equations describe the change in the amplitude of transverse vibrations of an elastic body during the transition through resonance at the main frequency of longitudinal vibrations at different speeds of motion of CFHM, provided that the nonlinear elastic properties of the body material satisfy the nonlinear technical law of elasticity, and the resistance force is proportional to the speed of portable motion in the power of $s_{1}$ :

$$
\begin{gathered}
\frac{d a}{d t}=\bar{k}_{1}(a)^{s_{1}}-\frac{b}{4\left(m_{t}+m_{s}\right)} m_{s} V l \omega \frac{\kappa \pi^{2}}{l} \sin \phi \\
\frac{d \phi}{d t}=\left(\frac{\pi}{l}\right)^{2} \sqrt{\frac{E I}{m_{t}+m_{s}}}-\omega-\frac{2 m_{s} V^{2}}{m_{t}+m_{s}}\left(\frac{\pi}{l}\right)^{2}-\frac{3 \bar{k}_{2}}{32} a^{2}+\frac{b}{4\left(m_{t}+m_{s}\right) a} m_{s} V l \omega \frac{\kappa \pi^{2}}{l} \cos \phi
\end{gathered}
$$

where $a$ is the amplitude of the main internal resonant vibrations.

As for the subresonances that occur when the condition is met $\kappa=2 \mathrm{~s}$, they are described by dependencies. 


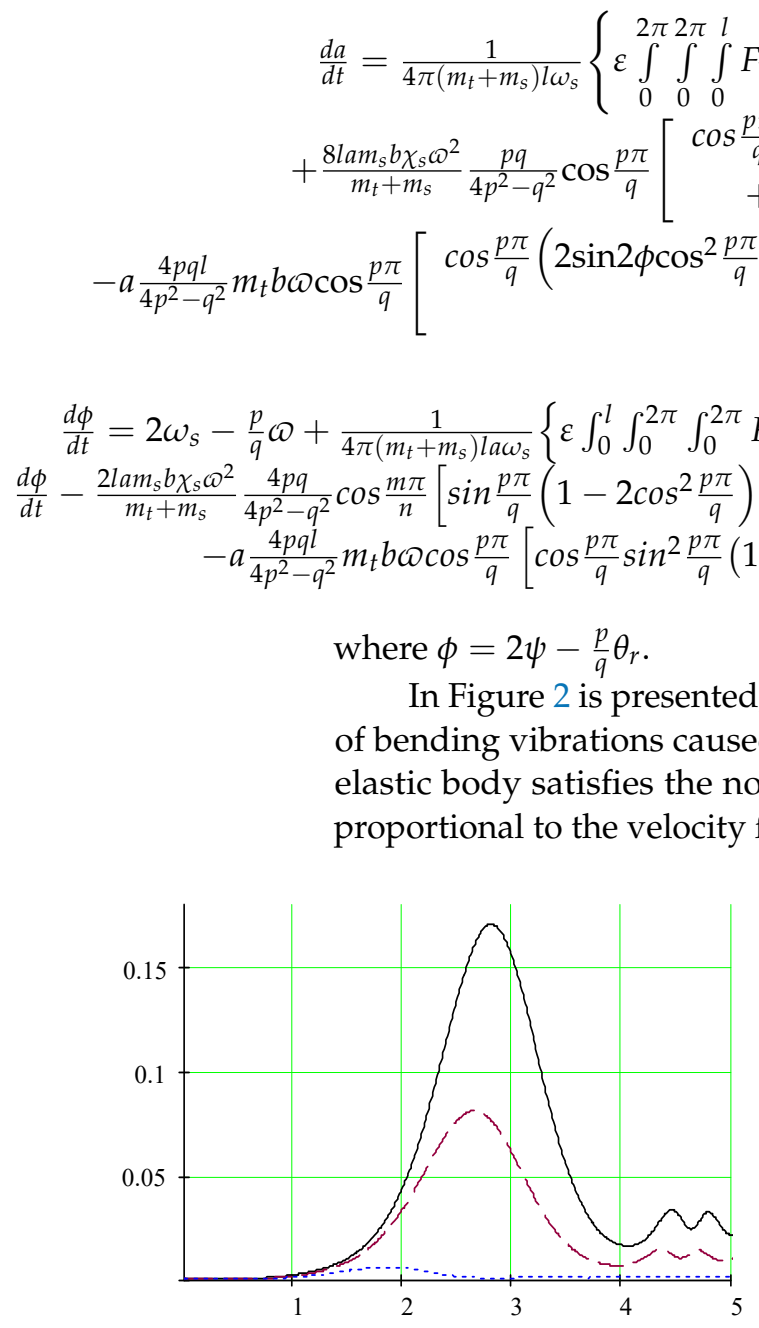

(a)

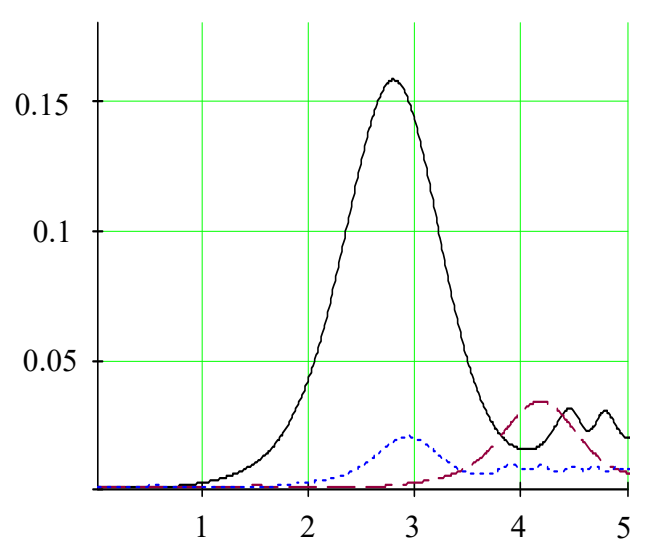

(c)

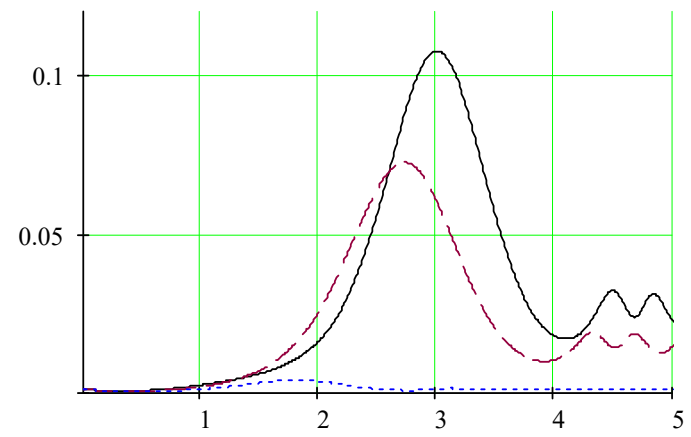

(b)

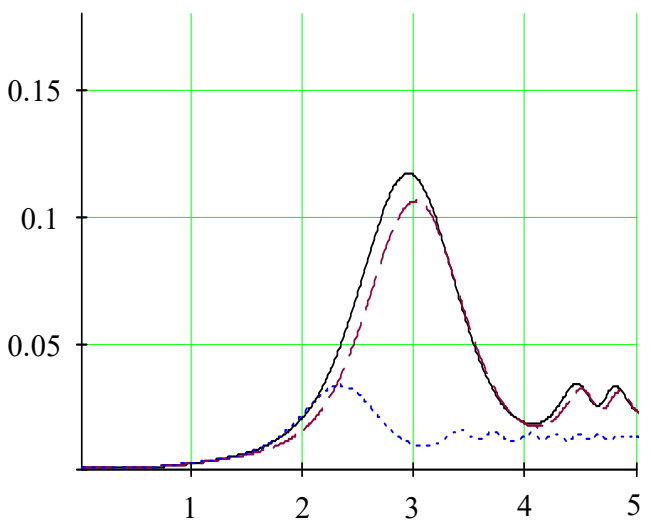

(d)

Figure 2. Laws of changes in the amplitude of bending vibrations during the transition through internal resonance at different values of the relative amount of motion CFHM $\left(k=\mathrm{m}_{\mathrm{s}} \mathrm{V}\right):(\mathbf{a})---k=200 \frac{\mathrm{kg}}{\mathrm{s}} \mathrm{m},---k=150 \frac{\mathrm{kg}}{\mathrm{s}} \mathrm{m}$, $-k=50 \frac{\mathrm{kg}}{\mathrm{s}} \mathrm{m}, m_{t}=30 ;(\mathbf{b})-\cdots-k=400 \frac{\mathrm{kg}}{\mathrm{s}} \mathrm{m},----k=300 \frac{\mathrm{kg}}{\mathrm{s}} \mathrm{m},-k=100 \frac{\mathrm{kg}}{\mathrm{s}} \mathrm{m}, m_{t}=30 ;(\mathbf{c})-\cdots$

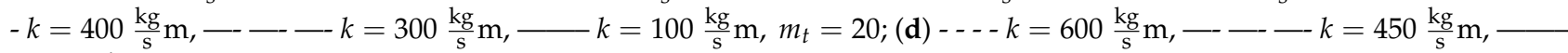
$k=10 \frac{\mathrm{kg}}{\mathrm{s}} \mathrm{m}, m_{t}=20$. 
In Figure 3a, a change in the amplitude of bending vibrations caused by longitudinal vibrations at different values of amplitudes can be observed, while in Figure $3 \mathrm{~b}$ is shown the motion of CFHM at different values of the speed of its movement.

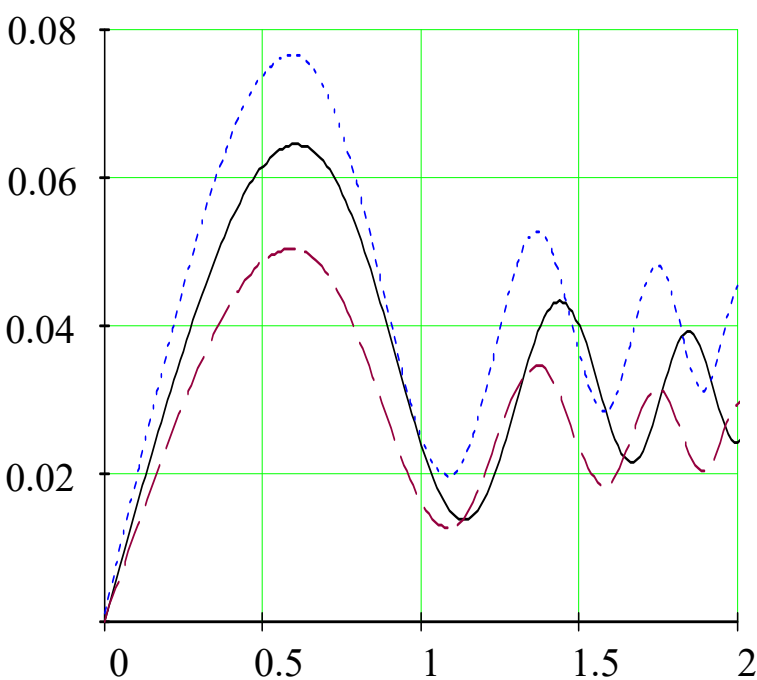

(a)

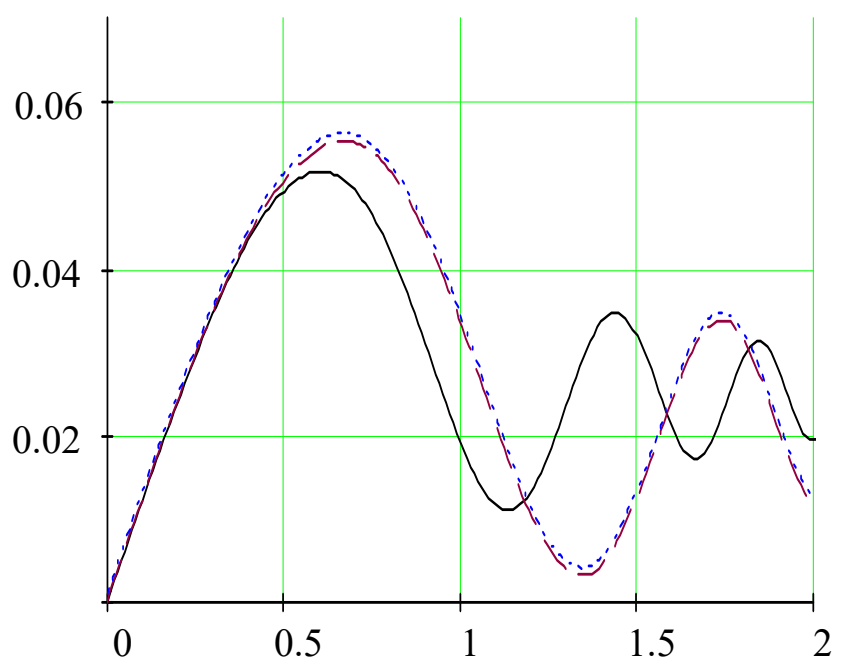

(b)

Figure 3. Change in the amplitude of bending vibrations during the transition through internal resonance at different values of the amplitude of longitudinal vibrations (a) $(---b=0.0015 \mathrm{~m},---b=0.001 \mathrm{~m},-b=0.0125 \mathrm{~m})$; and different speeds of motion CFHM (b) $(---V=1 \mathrm{~m} / \mathrm{s},---V=1.25 \mathrm{~m} / \mathrm{s},-V=5 \mathrm{~m} / \mathrm{s})$.

By analyzing the analytical dependences obtained above, it is established that internal resonances for an elastic body that performs bending and longitudinal vibrations can exist on even and odd modes of bending vibrations, and on even modes they are caused by longitudinal vibrations, and on odd modes by the motion CFHM. As for the quantitative characteristics of the internal resonance, the given graphical dependences show that the amplitude of the transition through the internal resonance is less:

- at higher frequencies of longitudinal vibrations than at the main one;

- for large values of the relative amount of motion CFHM;

- with a faster transition through resonance.

The above represented results have a practical component; in order to reduce dynamic loads in an elastic body that performs longitudinal and bending vibrations and along which a continuous stream motion of CFHM, it is necessary to move the latter at the highest technologically possible speed. At the same time, a special case mentioned above, $m_{s}=0$, is the result concerning resonant bending vibrations caused only by longitudinal vibrations. They, as follows from relations (34), exist only on paired modes of bending vibrations.

The main idea of studying internal resonant phenomena in the elastic body-CFHM system described above has been transferred to the study of external resonant phenomena. Below, we give only the basic relations concerning the continuous action of an external periodic perturbation, and the case of a discrete periodic (pulsed) action on the system under consideration is considered in more detail, because it requires some additional mathematical calculations.

Therefore, the condition for the main external resonance under the continuous action of an external perturbation is $\omega_{1} \approx \mu$ The relations that describe the resonant process at the main frequency of natural vibrations take the form 


$$
\begin{gathered}
a \frac{\partial \bar{B}(a, \bar{\phi})}{\partial \phi}\left(\omega_{1}-\mu\right)+2 \bar{A}(a, \bar{\phi}) \omega_{1}=\frac{1}{2\left(m_{t}+m_{s}\right) l \pi} \int_{0}^{l} \int_{0}^{2 \phi} F(a, y, \bar{\phi}+\theta, \theta) F \sin \frac{\pi}{l} y \sin (\bar{\phi}+\theta) d y d \theta \\
+\frac{1}{2\left(m_{t}+m_{s}\right) l \pi} \int_{0}^{l} \int_{0}^{2 \phi} F(a, y, \bar{\phi}+\theta, \theta) F \sin \frac{\pi}{l} y \cos (\bar{\phi}+\theta) d y d \theta
\end{gathered}
$$

where $\bar{\phi}$ is the relations that describe the resonant process at the main frequency of natural vibrations that take the form of the phase difference between natural and external forced vibrations, i.e., $\bar{\phi}=\psi-\theta$, so functions $\bar{A}(a, \bar{\phi})$ and $\bar{B}(a, \bar{\phi})$ describe the laws of change in the resonant parameters of the system under study using relations similar to (29), with the only difference that the resonant process is considered at the frequency of external perturbation.

In the specific case considered for non-resonant vibrations under the condition of external periodic perturbation, the system of Equation (35) is transformed to the form

$$
\begin{gathered}
a \frac{\partial \bar{B}(a, \bar{\phi})}{\partial \phi}\left(\omega_{1}-\mu\right)+2 \bar{A}(a, \bar{\phi}) \omega_{1}=v a^{s_{1}}+\frac{H}{2\left(m_{t}+m_{s}\right) \omega l \pi} \overline{\cos \phi}, \\
\frac{\partial \bar{A}(a, \bar{\phi})}{\partial \phi}(\omega-\mu)-2 a \omega \bar{B}(a, \bar{\phi})=a V^{2} \frac{m_{s}}{m_{t}+m_{s}}\left(\frac{\pi^{2}}{l}\right)-\frac{3 \varepsilon}{32} \frac{\pi^{2} \overline{k_{2}}}{l^{2}} \frac{a^{2}}{\omega_{1}}-\frac{H}{2\left(m_{t}+m_{s}\right) a \omega l \pi} \sin \bar{\phi},
\end{gathered}
$$

where $H$ is the value of the external sinusoidal periodic perturbation.

The differential dependences obtained above also allow us to obtain relations for determining the amplitude of stationary resonant vibrations

$$
\begin{gathered}
\frac{\beta}{m+m_{1}}(\bar{\omega})^{s_{1}-1} a^{s_{1}}-\frac{2 \varepsilon \bar{H}}{\pi(\bar{\omega}+v(t))} \cos \phi=0, \\
\bar{\omega}-\mu(t)-\left(\frac{\pi}{l}\right)^{2} \frac{m_{s}}{m_{t}+m_{s}} \frac{V^{2}}{8 \omega}=\varepsilon\left(\frac{2 \bar{H}}{\pi(\bar{\omega}+\mu(t)) a} \sin \phi+\frac{3 \bar{k}_{2}}{32} \frac{\pi^{2}}{l^{2}} \frac{a^{2}}{\omega}\right)
\end{gathered}
$$

By simple transformations from dependencies (37), we obtain a resonant curve that describes the dependence of the amplitude of stationary resonant vibrations on the unbalance of the frequencies of natural and forced vibrations and the parameters of the system

$\left(\frac{2 \varepsilon \bar{H}}{\pi(\omega+\mu(t))}\right)^{2}=\left(\frac{\beta}{m_{t}+m_{s}}(\bar{\omega})^{s_{1}-1} a^{s_{1}}\right)^{2}+a^{2}\left(\bar{\omega}-\mu(t)-\left(\frac{\pi}{l}\right)^{2} \frac{m_{s}}{m_{t}+m_{s}} \frac{V^{2}}{8 \bar{\omega}}-\frac{3 \bar{k}_{2} \varepsilon}{32} \frac{\pi^{2}}{l^{2}} \frac{a^{2}}{\omega}\right)^{2}$

Based on ratio (38), Figure 4 presents the dependencies of the amplitude of resonant stationary vibrations of an elastic body on the unbalance $\alpha=\omega-\mu$ of the frequencies of natural and forced vibrations at different speeds of motion of CFHM.

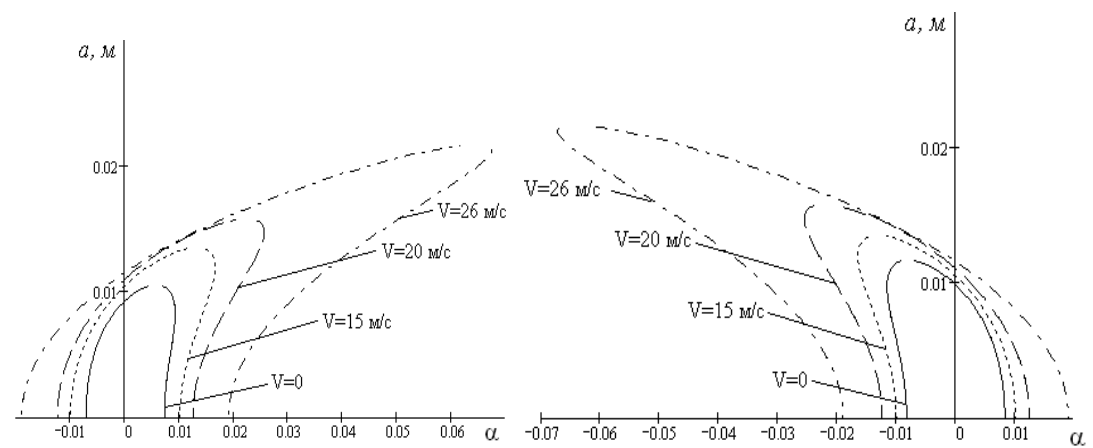

Figure 4. Dependences of the resonant amplitude of vibrations of an elastic body on the frequency unbalance at different values of the nonlinear component of the reducing force: $k_{2}<0, k_{2}>0$.

It follows from the above that when the sign of the nonlinear component of the reducing force changes, the slope of the resonant curves changes. Comparing the graphical dependencies for different models of the resistance force, it can be argued that an increase in the degree of its nonlinearity leads to a decrease in the resonant value of the vibration amplitude and a narrowing of the resonant zone.

As for the unknown function $U_{1}\left(a_{1}, y, \psi_{1}, \gamma, \theta\right)$, and for non-resonant or resonant cases, it partially affects only the change in the shape of bending vibrations and can be found without much difficulty by decomposing into multiple Fourier series, followed by 
equating the coefficients in the right and left parts of the relations that follow from (24) and (25), for the non-resonant case, and (31) and (36) for the resonant one. The case of pulsed (discrete) action of an external disturbance is much more important and at the same time much more difficult to develop a research methodology. Below, we consider the case when the specified perturbation acts only at a fixed point of the elastic body and is repeated at regular intervals. As for the limitations concerning the specified external perturbation of the vibrations, the elastic body-continuous flow of CFHM, as for continuous periodic perturbation, the maximum value of the pulse perturbation is small compared to the maximum value of the reduced transverse force. Thus, the differential equation of the bending component of the elastic oscillations takes the following form:

$$
\begin{aligned}
\left(m_{t}+m_{s}\right) \frac{\partial^{2} w}{\partial t^{2}} & +E I \frac{\partial^{4} w}{\partial y^{4}} \\
& =\varepsilon\left\{f_{1}\left(w, \frac{\partial w}{\partial t}, \ldots, \frac{\partial^{3} w}{\partial y^{3}}\right)+\delta\left(y-y_{0}\right) \sum_{j=1} \hat{f}_{j}\left(w, \frac{\partial w}{\partial t}, \ldots, \frac{\partial^{3} w}{\partial y^{3}}\right) \delta(t-j T)\right\} \\
& +g\left(\frac{\partial w}{\partial y}, \frac{\partial^{2} w}{\partial y^{2}}, \frac{\partial^{2} w}{\partial y \partial t}, \frac{\partial u}{\partial t}\right),
\end{aligned}
$$

in which $y_{0}$ is the coordinate of the elastic body in which the periodic impulse force acts with the period $T$, and $\delta(\ldots)$ is a function of the corresponding argument. Regarding the following forms, $f_{1}\left(w, \frac{\partial w}{\partial t}, \ldots, \frac{\partial^{3} w}{\partial y^{3}}\right)$ and $\hat{f}_{j}\left(w, \frac{\partial w}{\partial t}, \ldots, \frac{\partial^{3} w}{\partial y^{3}}\right)$, we can consider them to be analytical. The form $g\left(\frac{\partial w}{\partial y}, \frac{\partial^{2} w}{\partial y^{2}}, \frac{\partial^{2} w}{\partial y \partial t}, \frac{\partial u}{\partial t}\right)$ takes into account the effect of longitudinal oscillations and CFHM on bending.

From the properties of delta functions [19-21], it follows that the maximum values of the periodic action of pulsed perturbation at time points $j T$ are equal $\hat{f}_{j}\left(w(t, y), \frac{\partial w(t, y)}{\partial t}, \ldots, \frac{\partial^{3} w(t, y)}{\partial y^{3}}\right)_{\substack{t \\ y=y_{0}}}=j T$. To generalize the above method of studying the effect of longitudinal vibrations and dynamics of CFHM on bending, primarily on the basis of the properties of completeness and orthonormal system of functions that describe the forms of natural bending vibrations of an elastic body, delta function from a linear variable $\delta\left(y-y_{0}\right)$ will be as follows: $\delta\left(y-y_{0}\right)=\sum_{j=1} c_{m} \sin \frac{m \pi}{l} y$, in which the modulus $c_{m}$ is as follows $c_{m}=\frac{2}{l} \int_{0}^{l} \delta\left(y-y_{0}\right) \sin \frac{m \pi}{l} y d y=\frac{2}{l} \sin \frac{m \pi}{l} y_{0}$. As for the time component of the pulse perturbation, the most interesting case of the considered drilling is the case when the perturbation has a period close to the period of its own bending oscillations. Hence, in the form presented below we will consider $T \approx \frac{2 \pi}{\omega_{1}}$ pulsed perturbations causing resonant bending vibrations. In this case, the temporal component of its action can be precisely represented [20] in the following form: $\delta(t-j T)=\cos \left(\omega_{1} t\right) \delta\left(t-j \frac{2 \pi}{\omega_{1}}\right)=\cos \theta \delta\left(\frac{\theta-\theta_{j}}{\omega_{1}}\right)$, $\theta_{j}=2 \pi j$. For simplicity, it is assumed that the first impulse action coincides with the initial moment of motion. Thus, the conducted partial sampling allows to present the initial mathematical model in a simpler form:

$$
\begin{aligned}
& \left(m_{t}+m_{s}\right) \frac{\partial^{2} w}{\partial t^{2}}+E I \frac{\partial^{4} w}{\partial y^{4}}=g\left(\frac{\partial w}{\partial y}, \frac{\partial^{2} w}{\partial y^{2}}, \frac{\partial^{2} w}{\partial y \partial t}, \frac{\partial u}{\partial t}\right) \\
& +\varepsilon\left\{f_{1}\left(w, \frac{\partial w}{\partial t}, \ldots, \frac{\partial^{3} w}{\partial y^{3}}\right)+\frac{2}{l} \sum_{m} \sin \frac{m \pi}{l} y_{0} \sin \frac{m \pi}{l} y \sum_{j=1} \hat{f}_{j}\left(w, \frac{\partial w}{\partial t}, \ldots, \frac{\partial^{3} w}{\partial y^{3}}\right) \cos \theta \delta\left(\frac{\theta-\theta_{j}}{\omega_{1}}\right)\right\} .
\end{aligned}
$$

Note that the procedures performed with delta functions do not violate the accuracy of the obtained mathematical model in the form of relation (40) compared to the original (differential Equation (39)); in addition, to operate with the right part of the latter equation is much easier than the original. The use of the developed methodology for the study of resonant oscillations due to external periodic action for the boundary value problem (40), (10) allows the relationship of the main resonance to be represented as follows: 


$$
\begin{gathered}
a \frac{\partial \bar{B}(a, \bar{\phi})}{\partial \phi}\left(\omega_{1}-\frac{2 \pi}{T}\right)+2 \bar{A}(a, \bar{\phi}) \omega_{1}=\bar{f}_{1}(a)+ \\
\frac{1}{2\left(m_{t}+m_{s}\right) l \pi} \sum_{m} \sin \frac{m \pi}{l} y_{0} \sum_{j} \int_{0}^{l} \int_{0}^{2 \phi} \sin \frac{m \pi}{l} y F_{j}(a, y, \bar{\phi}+\theta) \cos \theta \delta\left(\frac{\theta-\theta_{j}}{\omega_{1}}\right) \sin \frac{\pi}{l} y \sin (\bar{\phi}+\theta) d y d \theta \\
\frac{\partial \bar{A}(a, \bar{\phi})}{\partial \phi}\left(\omega_{1}-\frac{2 \pi}{T}\right)-2 a \omega_{1} \bar{B}(a, \bar{\phi}) \\
=\widetilde{f}_{1}(a)+a V^{2} \frac{m_{s}}{m_{t}+m_{s}}\left(\frac{\pi^{2}}{l}\right) \\
+\frac{1}{2\left(m_{t}+m_{s}\right) l \pi} \sum_{m} \sin \frac{m \pi}{l} y_{0} \sum_{j} \int_{0}^{l} \int_{0}^{2 \phi} \sin \frac{m \pi}{l} y F_{j}(a, y, \bar{\phi} \\
+\theta) \cos \theta \delta\left(\frac{\theta-\theta_{j}}{\omega_{1}}\right) \sin \frac{\pi}{l} y \cos (\bar{\phi}+\theta) d y d \theta
\end{gathered}
$$

The system of differential equations is the basis for the study in the general case of the influence of impulse perturbation on the bending vibrations of the system continuous flow of CFHM. It is greatly simplified in the case where the impulse perturbation is constant, i.e., $f_{j}\left(w, \frac{\partial w}{\partial t}, \ldots, \frac{\partial^{3} w}{\partial y^{3}}\right) \equiv F_{0}=$ const. For this case, the basic equations for the main resonance in the case when the elastic properties of the body are described by the nonlinear technical law of elasticity, and the force of resistance is proportional to the velocity to the degree they take the form

$$
\begin{gathered}
a \frac{\partial \bar{B}(a, \bar{\phi})}{\partial \phi}\left(\omega_{1}-\frac{2 \pi}{T}\right)+2 \bar{A}(a, \bar{\phi}) \omega_{1}=v a^{s_{1}}+\frac{T F_{0}}{\left(m_{t}+m_{s}\right) \omega l} \sin \frac{\pi y_{0}}{l} \sum_{j=1} \operatorname{cosj} \overline{\cos \bar{\phi}} \\
\frac{\partial \bar{A}(a, \bar{\phi})}{\partial \phi}\left(\omega_{1}-\frac{2 \pi}{T}\right)-2 a \omega \bar{B}(a, \bar{\phi})=a V^{2} \frac{m_{s}}{m_{t}+m_{s}}\left(\frac{\pi^{2}}{l}\right)- \\
\frac{3 k_{2} \varepsilon}{32} \frac{\pi^{2}}{l^{2}} \frac{a^{2}}{\omega_{1}}-\frac{T F_{0}}{2\left(m_{t}+m_{s}\right) a \omega l \pi} \sin \frac{\pi y_{0}}{l} \sum_{j=1} \operatorname{cosj} \overline{\sin \bar{\phi}}
\end{gathered}
$$

For the first resonant approximation, the system of differential Equation (42) describes the main parameters of resonant vibrations under the action of external periodic pulse perturbation by the following dependence:

$$
\begin{gathered}
\frac{d a}{d t}==v a^{s_{1}}+\frac{T F_{0}}{\left(m_{t}+m_{s}\right) \omega l} \sin \frac{\pi y_{0}}{l} \sum_{j=1} \operatorname{cosj} T \cos j T \overline{\cos \bar{\phi}}, \\
\frac{d \bar{\phi}}{d t}=\omega_{1}-\frac{2 \pi}{T}-a V^{2} \frac{m_{s}}{m_{t}+m_{s}}\left(\frac{\pi^{2}}{l}\right)-\frac{3 k_{2} \varepsilon}{32} \frac{\pi^{2}}{l^{2}} \frac{a^{2}}{\omega_{1}}-\frac{T F_{0}}{2\left(m_{t}+m_{s}\right) a \omega l \pi} \sin \frac{\pi y_{0}}{l} \sum_{j=1} \cos j T \overline{\sin \bar{\phi}} .
\end{gathered}
$$

According to the above dependence, Figure 5 shows the change in the amplitude of bending vibrations during the transition through resonance at different lengths of the elastic body, the quantities of the relative motion of the CFHM, and the points of application of impulse perturbation. 


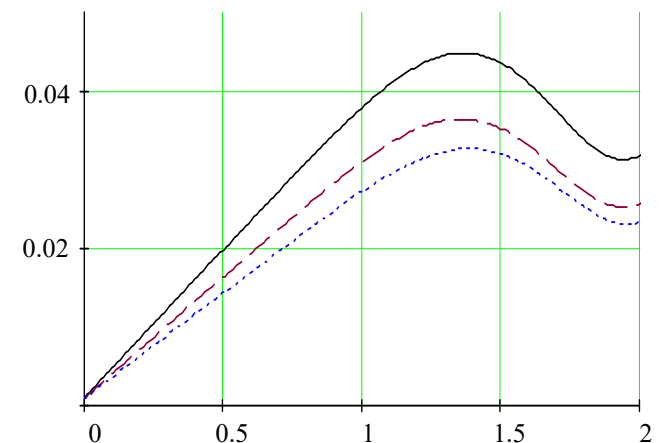

(a)

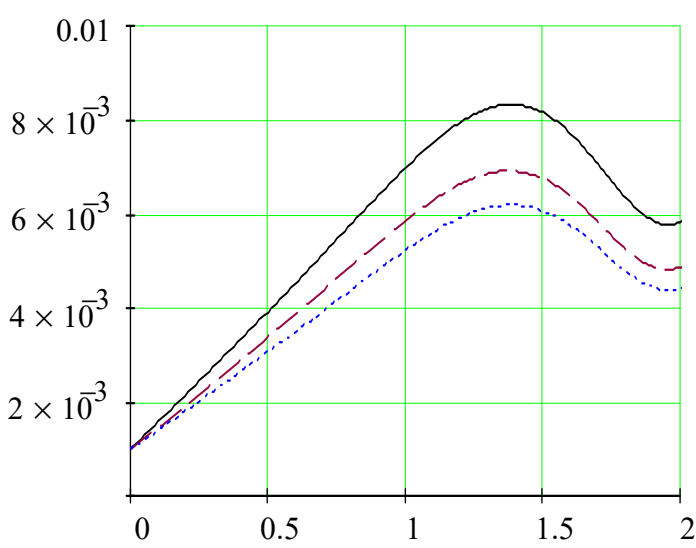

(c)

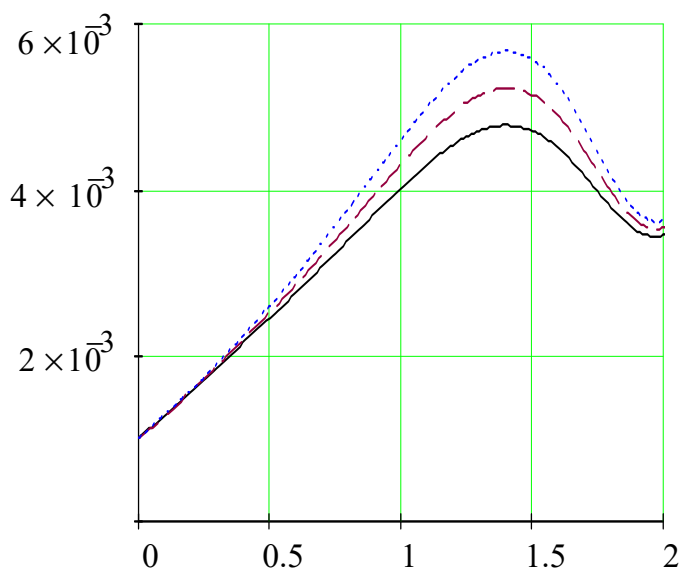

(e)

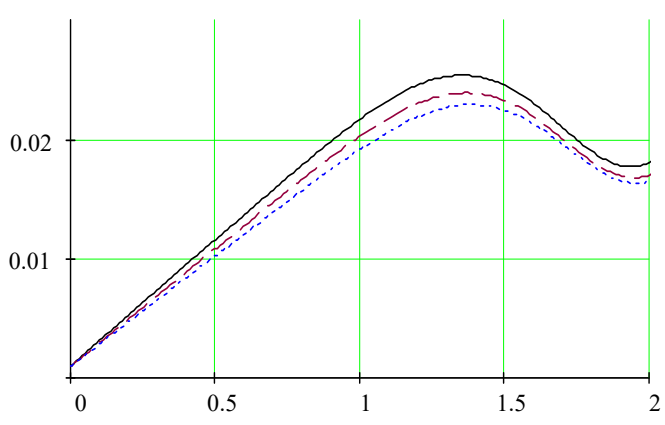

(b)

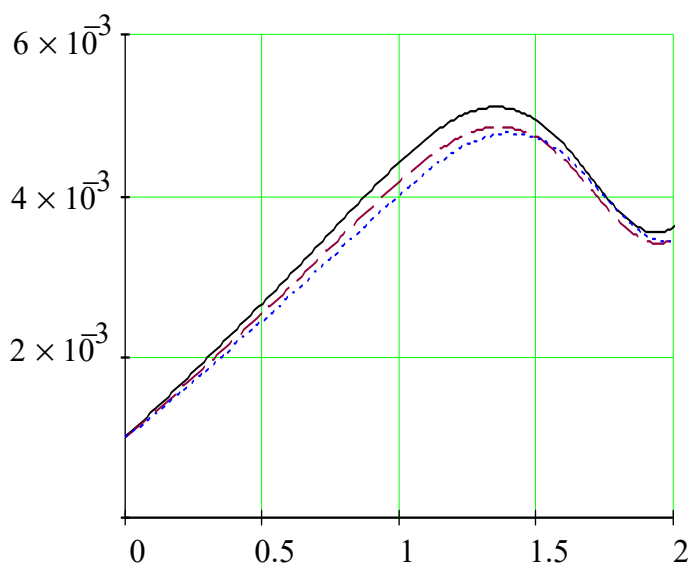

(d)

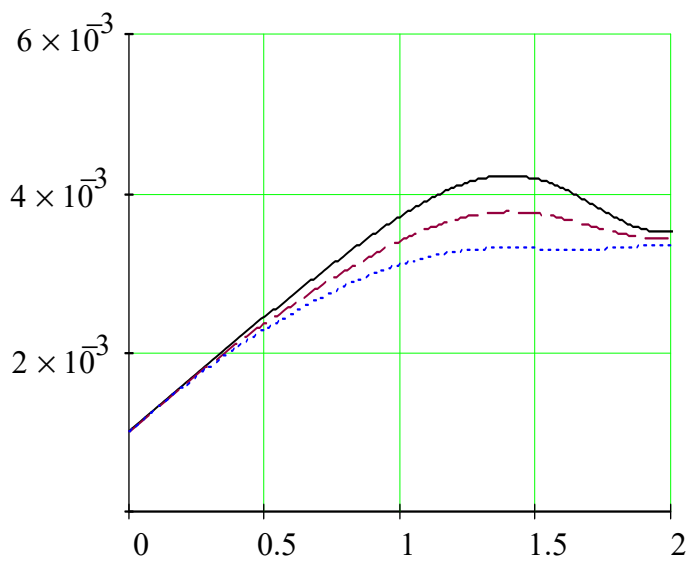

(f)

Figure 5. Time change of the amplitude of resonant vibrations due to periodic pulse action at different points of application. (a) - $k=400 \frac{\mathrm{kg}}{\mathrm{s}} \mathrm{m},-\longrightarrow-k=300 \frac{\mathrm{kg}}{\mathrm{s}} \mathrm{m},-k=100 \frac{\mathrm{kg}}{\mathrm{s}} \mathrm{m}, y_{0}=\frac{1}{4}, l=8 \mathrm{~m} ;(\mathbf{b})-k=400 \frac{\mathrm{kg}}{\mathrm{s}} \mathrm{m},-\longrightarrow-\longrightarrow$ $k=300 \frac{\mathrm{kg}}{\mathrm{s}} \mathrm{m}, \longrightarrow k=100 \frac{\mathrm{kg}}{\mathrm{s}} \mathrm{m}, y_{0}=\frac{1}{4}, l=6 \mathrm{~m} ;(\mathrm{c})-k=600 \frac{\mathrm{kg}}{\mathrm{s}} \mathrm{m},---k=450 \frac{\mathrm{kg}}{\mathrm{s}} \mathrm{m},-k=150 \frac{\mathrm{kg}}{\mathrm{s}} \mathrm{m}, y_{0}=$ $\frac{1}{6}, l=8 \mathrm{~m} ;(\mathbf{d})-k=600 \frac{\mathrm{kg}}{\mathrm{s}} \mathrm{m},---k=450 \frac{\mathrm{kg}}{\mathrm{s}} \mathrm{m},-k=150 \frac{\mathrm{kg}}{\mathrm{s}} \mathrm{m}, y_{0}=\frac{1}{6}, l=6 \mathrm{~m} ;(\mathbf{e})-k=600 \frac{\mathrm{kg}}{\mathrm{s}} \mathrm{m}$,

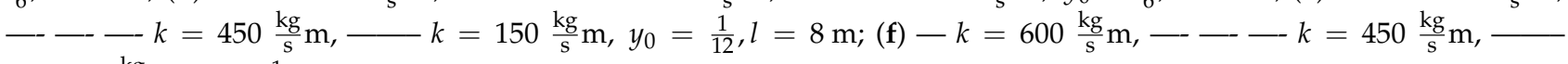
$\left.k=150 \frac{\mathrm{kg}}{\mathrm{s}} \mathrm{m}, y_{0}=\frac{1}{12}, l=6 \mathrm{~m}\right)$.

The presented graphical dependencies show the amplitude of the passage through the main resonance of the bending vibrations of the elastic body, which is due to the 
periodic pulse action. It becomes more important in the case when the point of application of this action is closer to the middle of the elastic body. As for the continuous flow of a homogeneous medium, for larger values of the relative amount of motion, it not only reduces the natural frequency of the elastic body but also slightly reduces the maximum value of the amplitudes of the system through the resonance.

The above results can be generalized without much difficulty in the case of the action of impulse perturbation at several points of an elastic body.

\section{Conclusions}

A methodological study of complex vibrations has been developed for the mechanical system of an elastic body-continuous flow of homogeneous medium (CFHM). The methodology is based on:

(a) the use of a priori information on some of the vibrations forms;

(b) the construction of a "simplified" mathematical model of system dynamics, which provides a wide range of external and internal factors and information on the longitudinal vibrations of an elastic body;

(c) obtaining analytical relationship that describe the overall range of factors on the elastic vibrations of system.

Considering the above given information, it allows us to conclude the following:

- CFHM motion affects both the natural frequency of bending and longitudinal vibrations;

- the most dangerous resonance vibrations of the specified systems type can be caused both by external periodic forces and mutual influence of one form of vibrations on the others (internal resonances);

- internal resonances for an elastic body that performs bending and longitudinal vibrations can exist in even and odd modes of bending vibrations, and they are caused by longitudinal vibrations in even modes, and by continuous flow of homogeneous medium motion in odd modes;

- the amplitude of the bending vibrations of the transition through the internal resonance at higher longitudinal vibrations frequencies is less than at the second frequency;

- the amplitude of the transition through the main resonance for larger values of the amount of CFHM motion is smaller than the amplitude of the transition during the rapid transition through the resonance.

The methodology can be used for other cases of complex vibrations of elastic bodies, and the obtained results can serve as a basis for choosing the main technological and operational parameters of elastic elements of mechanisms and machines that perform complex vibrations.

Practical use of the obtained results. The theoretical results obtained in this work can be the basis for calculating the following:

- the blast effect on the elements of protective structures in order to increase their protective capacity by improving the method of their attachment or by using additional reinforcement;

- buff load effects on the elements of drilling strings and dynamic processes that occur during surface strengthening by work hardening in order to avoid resonance phenomena;

- technological processes of vibration displacement or vibration separation of granular media;

- longitudinal frequencies of movement of rope lifts in order to avoid resonant phenomena in them, and thus to extend the resource to make the object;

- reducing dynamic performance bearings for mounting rotors; 
- $\quad$ assessing structural strength and vibration reduction of mechanical systems in energy, taking into account the optimal parameters (motion, mass, stiffness, etc.), which are found on the basis of empirical information and methodology developed in the work using interpretation and synthesis.

Author Contributions: Study analysis, A.A., S.F., and M.S.; methodology, A.A., S.F., and M.S.; resources, S.F., A.A., and M.S.; validation, S.F., A.A., and M.S.; writing—original draft preparation, Y.S., S.F., A.A., Y.K., and M.S.; writing-review and editing, Y.S., S.F., A.A., T.P. and M.S.; visualization, S.F., A.A. and M.S.; project administration, S.F., T.P. All authors have read and agreed to the published version of the manuscript.

Funding: This work was supported by the Slovak Research and Development Agency under the contract No. APVV-19-0581 and by of the Faculty of Management of Comenius University in Bratislava, Slovak Republic.

Institutional Review Board Statement: Not applicable.

Informed Consent Statement: Not applicable.

Conflicts of Interest: The authors declare no conflict of interest.

\section{References}

1. Bogolyubov, N.N.; Mitropolsky, Y.A. Asymptotic Methods in the Theory of Nonlinear Oscillations; Gordon and Breach Publishers: London, UK, 1958; p. 408.

2. Mitropolskii, Y.A.; Moseenkov, B.I. Asymptotic Solutions of Partial Differential Equations; Vyshcha Shkola: Kyiv, Ukraine, 1976.

3. Pukach, P.Y.; Kuzio, I.V. Resonance phenomena in quasi-zero stiffness vibration isolation systems. Sci. Bull. Natl. Min. Univ. 2015, 3, 62-67.

4. Yarka, U.; Fedushko, S.; Veselý, P. The Dirichlet Problem for the Perturbed Elliptic Equation. Mathematics 2020, 8, 2108. [CrossRef]

5. Goldin, A.S. Vibration of Rotary Machines; Mechanical Engineering: Moscow, Russia, 2000; p. 344.

6. Peleschyshyn, A.; Syerov, Y.; Tarasov, D.; Zhezhnych, P. Intelligent system of information gathering and analysis from news Internet-resources. In Proceedings of the Advanced Computer Systems and Networks: Design and Application (ACSN-2005), Lviv, Ukraine, 21-23 September 2005; pp. 48-52.

7. Ohorodnikov, P. Investigation of the relationship between longitudinal and torsional vibrations of the drill string. Oil Ind. Ukr. 2014, 2, 6-9.

8. Huliaiev, V.I.; Borshch, O.I. Spiral waves in swirling elastic quills rotating with internal fluid flow. Acoust. Bull. 2007, 10, 12-18.

9. Andrukhiv, A.; Sokil, B.; Sokil, M. Resonant phenomena of elastic bodies that perform bending and torsion vibrations. Ukr. J. Mech. Eng. Mater. Sci. 2018, 3, 65-73. [CrossRef]

10. Andrianov, I.V.; Danishevskyi, V.V.; Ivankov, A.O. Asymptotic Methods in the Theory of Vibrations of Beams and Plates; Dnipropetrovsk State Academy of Civil Engineering and Architecture: Dnipro, Ukraine, 2010; p. 217.

11. Gendelman, O.; Vakakis, A.F. Transitions from localization to nonlocalization in strongly nonlinear damped oscillators. Chaos Solitons Fractals 2000, 11, 1535-1542. [CrossRef]

12. Cveticanin, L. Period of vibration of axially vibrating truly nonlinear rod. J. Sound Vib. 2016, 374, 199-210. [CrossRef]

13. Cveticanin, L.; Pogany, T. Oscillator with a sum of non-integer order non-linearities. J. Appl. Math. 2012, 649050. [CrossRef]

14. Pukach, P.Y.; Kuzio, I.V.; Nytrebych, Z.M.; Ilkiv, V.S. Analytical methods for determining the effect of the dynamic process on the nonlinear flexural vibrations and the strength of compressed shaft. Sci. Bull. Natl. Min. Univ. 2017, 5, 69-76.

15. Pukach, P.Y.; Kuzio, I.V.; Nytrebych, Z.M.; Ilkiv, V.S. Asymptotic method for investigating resonant regimes of non-linear bending vibrations of elastic shaft. Sci. Bull. Natl. Min. Univ. 2018, 1, 68-73.

16. Chen, L.Q.; Zu, J.W.; Wu, J. Transverse vibrations of an axially accelerating viscoelastic string with geometric nonlinearity. J. Eng. Math. 2004, 48, 171-182. [CrossRef]

17. Chen, L.Q. Analysis and Control of Transverse Vibrations of Axially Moving Strings. Appl. Mech. Rev. 2005, 58, 91-116. [CrossRef]

18. Gevko, I.; Lyashuk, O.; Djachun, A.; Dovbush, A. Interpretation of the choice of conveyers with improved technological characteristics. MOTROL. In Commission of Motorization and Energetics in Agriculture; Polska Akademia Nauk: Lublin-Rzeszow, Poland, 2015; Volume 17, pp. 107-116.

19. Lyashuk, O.; Vovk, Y.; Sokil, B.; Klendii, V.; Ivasechko, R.; Dovbush, T. Mathematical model of a dynamic process of transporting a bulk material by means of a tube scraping conveyor. Agric. Eng. Int. CIGR J. 2019, 21, 74-81.

20. Sokil, M. Nonlinear vibrations of flexible tubular bodies, along which a continuous flow of medium moves. Collection of scientific and technical works. Sci. Bull. Natl. Min. Univ. 2014, 24, 351-356.

21. Sokil, B.; Senyk, A.; Sokil, M.; Andrukhiv, A.; Kovtonyuk, M.; Gromaszek, K.; Ziyatbekova, G.; Turgynbekov, Y. Mathematical models of dynamics of friable media and analytical methods of their research. Prz. Elektrotechniczny 2019, 95, 74-78. [CrossRef]

22. Kharchenko, Y.V.; Sokil, M.B. Vibrations of Moving Nonlinearly Elastic Media and Asymptotic Method in Their Study; Science Bulletin; Coll. Scientific and Technical Works: Lviv, Ukraine, 2006; Volume 16, pp. 134-138. 
23. Delta Function. Mathematics. Available online: https:/ / mathworld.wolfram.com/DeltaFunction.html (accessed on 17 July 2020).

24. Markovych, B.M. Equations of Mathematical Physics; Lviv Polytechnic Publishing House: Lviv, Ukraine, 2010 ; p. 384.

25. Oleynik, O.A. Lectures on Partial Differential Equations; Binomial: Moscow, Russia, 2005.

26. Sokil, B.I.; Khytriak, O.I. Inverse Problems of Dynamics on Vibrations of Media Described by a Perturbed Breserton Equation. Scientific Herald of the NLTU of Ukraine; Coll. Scientific and Technical Works: Lviv, Ukraine, 2010; Volume 20, pp. 290-296.

27. Goroshko, A.V.; Roizman, V.P. Statistical Methods for Providing the Stability of the Solutions of Inverse Problems and their Application to Decrease Rotor Vibroactivity. J. Mach. Manuf. Reliab. 2015, 44, 232-238. [CrossRef]

28. Ryabchuk, N.; Grishko, N.; Grishko, V.; Rudenko, A.; Petryk, V.; Bapiyev, I.; Fedushko, S. Artificial Intelligence Technologies Using in Social Engineering Attacks. Available online: http:/ / ceur-ws.org/Vol-2654/paper43.pdf (accessed on 19 August 2020)

29. Sokolov, G.; Syerov, Y. Building a Computer Model of an Acoustic Signal Recognition Device. Available online: http:/ / ceur-ws. org/Vol-2654/paper37.pdf (accessed on 19 August 2020).

30. Goroshko, A.V.; Royzman, V.P.; Barmina, O.V. Study of the Stability and Accuracy of the Eccentricity Identification Algorithm in Fast-Revolving Rotor Balancing Problems. J. Mach. Manuf. Reliab. 2016, 45, 227-232. [CrossRef]

31. Royzman, V.; Goroshko, A. Multiple inverse problem. J. Vibroeng. 2012, 14, 1417-1424. 